\title{
Direct profiling of genome-wide dCas9 and Cas9 specificity using ssDNA mapping (CasKAS)
}

\author{
Georgi K. Marinov ${ }^{1, \#}$, Samuel H. Kim², S. Tansu Bagdatli ${ }^{1}$, Alexandro E. Trevino ${ }^{3,4}$, Josh Tycko ${ }^{1}$, \\ Tong Wu ${ }^{5}$, Lacramioara Bintu ${ }^{4}$, Michael C. Bassik ${ }^{1,6}$, Chuan He ${ }^{5,7,8}$, Anshul Kundaje ${ }^{1,9}$, and William J. \\ GreEnLEAF $^{1,10,11,12, \#}$ \\ ${ }^{1}$ Department of Genetics, School of Medicine, Stanford University, Stanford, CA 94305, USA \\ ${ }^{2}$ Cancer Biology Program, School of Medicine, Stanford University, Stanford, CA 94305, USA \\ ${ }^{3}$ Center for Personal Dynamic Regulomes, Stanford University, Stanford, CA 94305, USA \\ ${ }^{4}$ Department of Bioengineering, Stanford University, Stanford, CA 94305, USA \\ ${ }^{5}$ Department of Chemistry, The University of Chicago, Chicago, IL, 60637, USA \\ ${ }^{6}$ Chemistry, Engineering, and Medicine for Human Health (ChEM-H), Stanford University, Stanford, CA, 94305, USA \\ ${ }^{7}$ Department of Biochemistry and Molecular Biology and Institute for Biophysical Dynamics, The University of Chicago, Chicago, \\ IL, 60637, USA \\ ${ }^{8}$ Howard Hughes Medical Institute, The University of Chicago, Chicago, IL, 60637, USA \\ ${ }^{9}$ Department of Computer Science, Stanford University, Stanford, CA 94305, USA \\ ${ }^{10}$ Department of Applied Physics, Stanford University, Stanford, CA 94305, USA \\ ${ }^{11}$ Center for Personal Dynamic Regulomes, Stanford University, Stanford, CA, 94305, USA \\ ${ }^{12}$ Chan Zuckerberg Biohub, San Francisco, California, USA \\ \# Corresponding author
}

Detecting and mitigating off-target activity is critical to the practical application of CRISPRmediated genome and epigenome editing. While numerous methods have been developed to map Cas9 binding specificity genome-wide, they are generally time-consuming and/or expensive, and not applicable to catalytically dead CRISPR enzymes. We have developed a rapid, inexpensive, and facile assay for identifying off-target CRISPR enzyme binding and cleavage by chemically mapping the unwound single-stranded DNA structures formed upon binding of a sgRNA-loaded Cas9 protein ("CasKAS"). We demonstrate this method in both in vitro and in vivo contexts.

CRISPR-based methods for editing the genome and epigenome have emerged as a highly versatile means of manipulating the genetic makeup and regulatory states of cells. CRISPR technologies hold the potential to transform medical practice by enabling direct elimination of pathogenic sequence variants or manipulation of aberrant gene expression programs. CRISPR has also become a standard tool for discovery in biomedical research, including its uses for high-throughput, massively parallel genomic screens ${ }^{1}$.

The presence of significant off-target effects is of universal concern for genome engineering technologies, presenting a major hurdle to fully realizing their potential utility. CRISPR tools have been shown to exhibit biochemical activity away from their intended target sites, which is partic- ularly problematic for therapeutic applications, where risks of activity at sites other than the intended target leading to negative consequences to patient health must be minimal. Understanding and mapping these effects is therefore an urgent need.

To this end, numerous experimental approaches have been developed to experimentally map off-target effects genome-wide. Methods such as Digenome-seq ${ }^{2]}$ look for particular types of cut sites around target sequences in whole-genome sequencing data; however, deep wholegenome sequencing remains expensive. Assays such as BLESS $^{3}$, GUIDE-seq ${ }^{4}$, HTGTS $^{15}$, DSBCapture ${ }^{6}$, BLISS ${ }^{17}$, SITE-seq ${ }^{8}$, CIRCLE-seq ${ }^{9}$, TTISS 10 , INDUCE-seq 11 , and CHANGE-seq ${ }^{12}$ aim instead to directly map Cas9 cleavage events. However, all these methods involve some combination of complex and laborious molecular biology protocols and non-standard reagents, and have not been widely adopted. Other methods, such as DISCOVER-seq $\frac{13}{13}$, which maps DNA repair activity by applying ChIP-seq against the MRE11 protein, as well as earlier applications of ChIP-seq to map catalytically dead dCas9 occupancy sites genomewide ${ }^{14 \mid 15}$, suffer from technical issues associated with the ChIP procedure. Most recently, long-read sequencing has been adapted to the problem of Cas9 specificity profiling, in the form of SMRT-OTS and Nano-OTS $\frac{16}{16}$, but the cost of these methods is relatively high while their throughput is comparatively low. 
bioRxiv preprint doi: https://doi.org/10.1101/2021.04.16.440202; this version posted April 17, 2021. The copyright holder for this preprint (which was not certified by peer review) is the author/funder, who has granted bioRxiv a license to display the preprint in perpetuity. It is made available under aCC-BY-NC-ND 4.0 International license.

a

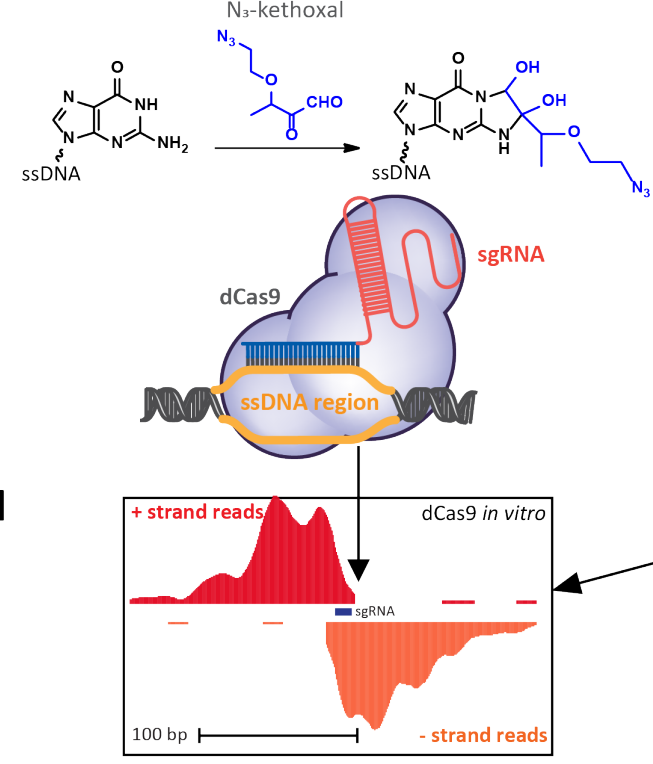

b
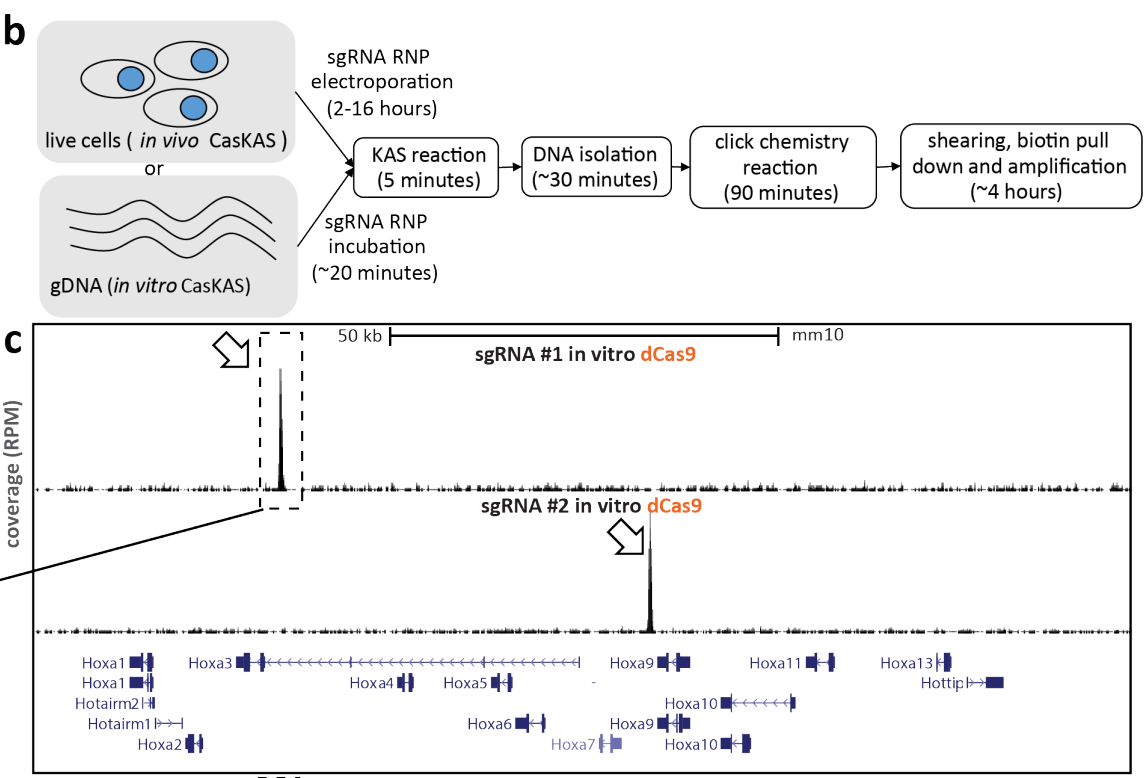

f
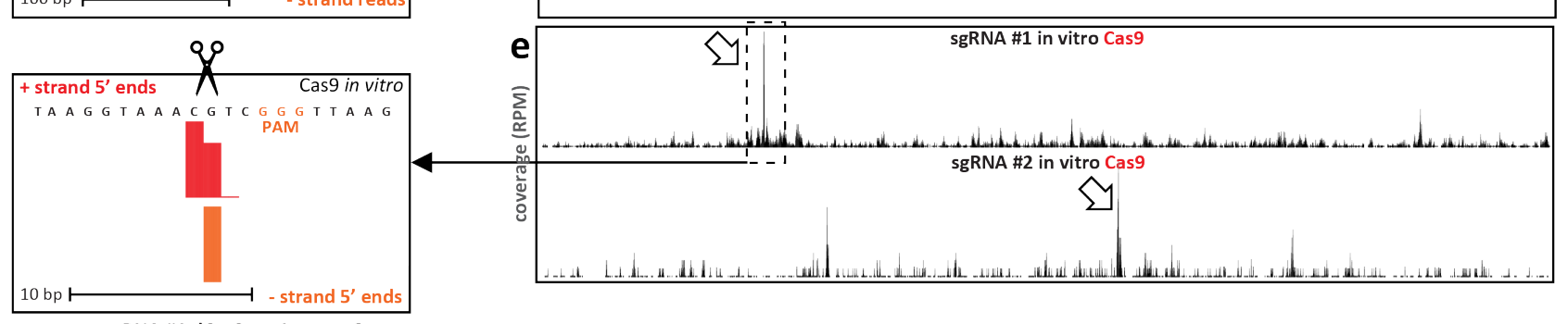

g

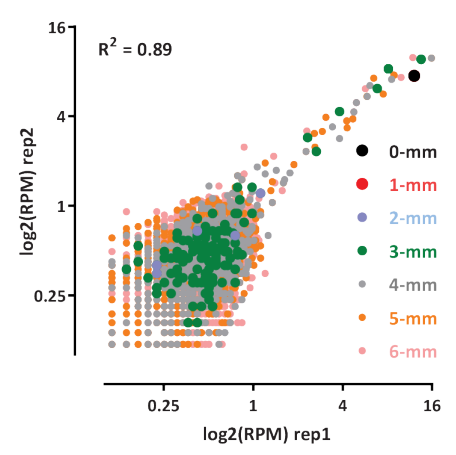

h

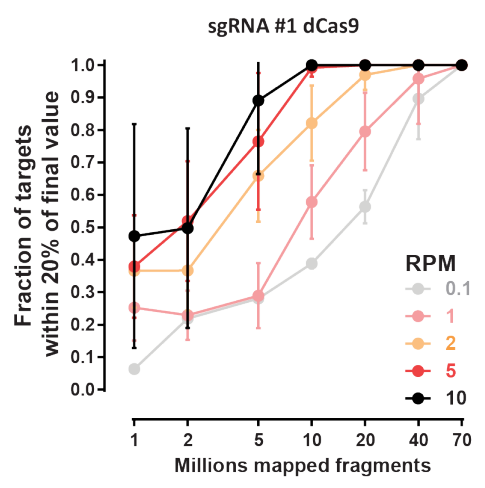

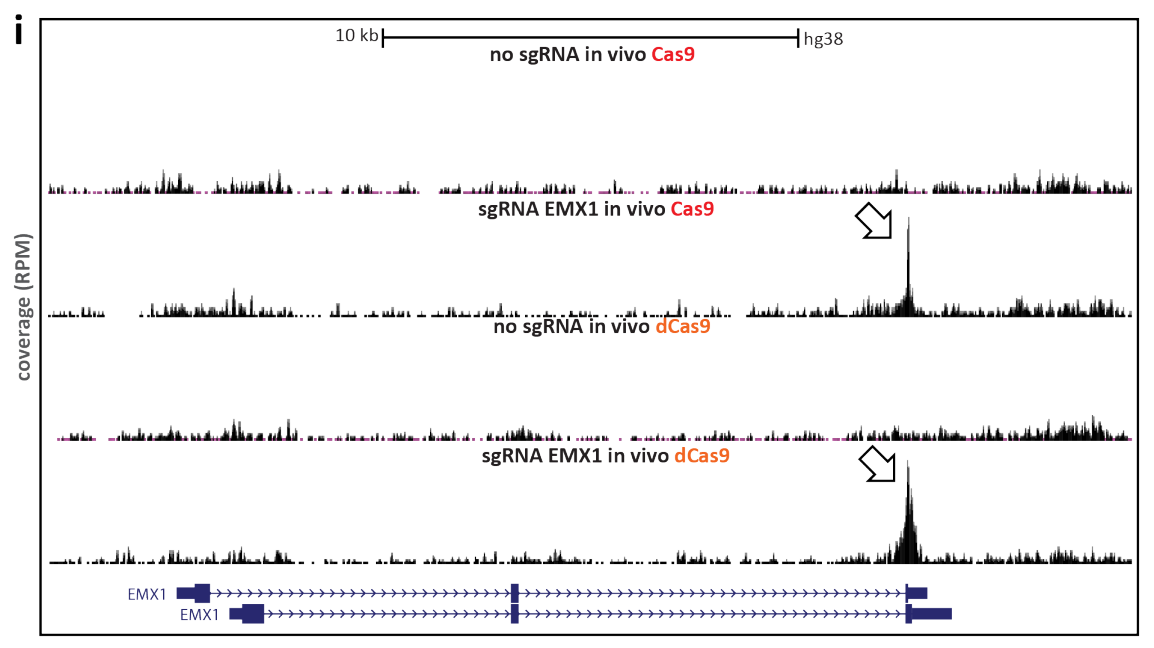
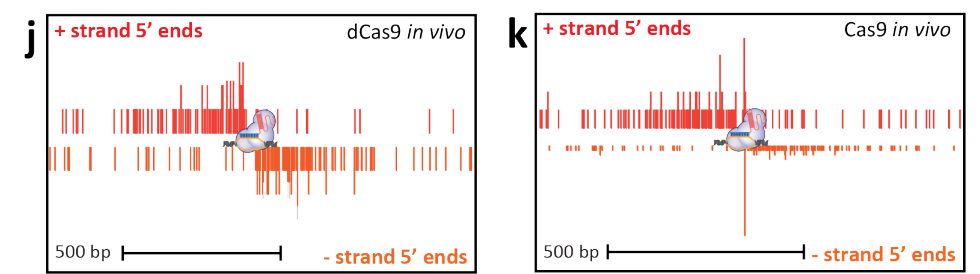

Figure 1: CasKAS maps dCas9- and Cas9-mediated strand invasion and cleavage events genome-wide in vitro and in vivo. (a) CasKAS is based on the KAS-seq assay for mapping ssDNA structures. $\mathrm{N}_{3}$-kethoxal covalently modifies unpaired guanine bases (while having no activity for G bases paired within dsDNA). Strand invasion by Cas9/dCas9 carrying an sgRNA results in the formation of a ssDNA structure, which can be directly identified 
Various computational models have also been trained to predict off-targets genome-wide ${ }^{17 / 18}$. However, these exhibit far from perfect accuracy, and thus in many situations, especially within clinical contexts, direct experimental evidence is needed to accurately identify potential unintended effects of CRISPR-based reagents.

A faster, more accessible, and versatile method for mapping CRISPR off targets is thus still a critical need in the field. When a Cas9-sgRNA ribonucleoprotein (RNP) is engaged with its target site, the sgRNA invades the DNA double helix, forming a ssDNA structure on the other strand (Fig. 1 1a). We thus reasoned that mapping ssDNAcontaining regions should be a sensitive biochemical signal of productive Cas9 binding. The recently developed KAS-seq ${ }^{[19}$ assay for mapping single-stranded DNA (ssDNA) (kethoxal-assisted ssDNA sequencing ${ }^{19}$ ) is ideally suited for the purpose of identifying ssDNA generated by CRISPR protein binding to DNA (Fig. 1 $1 \mathrm{a}-\mathrm{b}$ ). KAS-seq is based on the specific covalent labeling of unpaired guanine bases with $\mathrm{N}_{3}$-kethoxal, generating an adduct to which biotin can then be added using click chemistry. After shearing, biotinylated DNA, corresponding to regions containing ssDNA structure, can be specifically enriched for and sequenced.

To determine the feasibility of using KAS-seq to map regions of ssDNA generated by Cas9 binding, we carried out an initial in vitro experiment using mouse genomic DNA (gDNA), purified dCas9 and two sgRNAs targeting the Hoxa locus.

Strikingly, we observed strong peaks at the expected target sites for each sgRNA (Fig. 1 1 ). Detailed examination of dCas9 CasKAS profiles around the predicted sgRNA target sites revealed strand coverage asymmetry patterns similar to those observed for ChIP-seq around transcription factor binding sites ${ }^{20}$ (Fig. 11d), indicating that enrichment derives from the sgRNA target site itself and confirming the utility of $\mathrm{N}_{3}$-kethoxal for mapping dCas9 occupancy sites. We termed the assay "CasKAS".

We then reasoned that CasKAS should also capture active Cas9 complexed with DNA, as the enzyme is thought to remain associated with DNA for some time after cleavage 21. We performed CasKAS experiments with the same sgRNAs and active Cas9 nuclease, and again observed enrichment at the expected on-target sites (Fig. 11). Examination of Cas9 CasKAS read profiles around the on-target site showed that the 5' ends of reads are precisely positioned around the expected cut site, with one cut position on the target strand (which binds the sgRNA and is cleaved by the HNH domain) and two to three such positions on the non-target strand (which is cleaved by the RuvC domain; Fig. 1:), consistent with the previously known patterns of Cas9 cleavage $e^{22123}$. CasKAS therefore provides target specificity profiles for both active and catalytically dead Cas9 enzymes.

In vitro CasKAS data was highly reproducible between replicates (Fig. 1 $\mathrm{g}$ ), and a modest sequencing depth of between 10 and 20 million mapped reads was sufficient to capture off-target specificity profiles (Fig. 1h), which is an order of magnitude lower than required for resequencing the whole genome.

We observed similar results with two mouse sgRNAs targeting the Nanog locus (Supplementary Fig. 1) and with two human sgRNA ("EMX1" and "VEGFA"; Supplementary Fig. 2 and 3). We found no enrichment using components of the RNP in isolation - sgRNAs, dCas9 or Cas9 (Supplementary Fig. 2).

Next we tested the application of CasKAS in vivo. Living cells contain substantial ssDNA due to active transcription and other processes ${ }^{19}$, so in vivo CasKAS signal derives from a mixture of Cas9-associated ssDNA and endogenous processes. We carried out KAS-seq experiments using both dCas9 and Cas9 in HEK293 cells transfected with RNPs targeting EMX1 or VEGFA, as well as negative, no-guide controls, which provided a map of background endogenous ssDNA profiles. At EMX1, which is not active in HEK293 cells, we observe strong peaks at the expected target site (Fig. 1.), as well as an asymmetric read profile around it for dCas9 (Fig. 1 1j), and a substantial degree of 5' end clustering at the cut site, similar to what is observed in vitro for active Cas9 (Fig. 1 $\mathrm{g}$ ). The VEGFA gene is active in

using $\mathrm{N}_{3}$-kethoxal. (b) Outline of in vivo and in vitro CasKAS. For in in vitro CasKAS, gDNA is incubated with a dCas9/Cas9 RNP, then $\mathrm{N}_{3}$-kethoxal is added to the reaction; for in in vivo CasKAS, cells are transfected with an RNP, then treated with kethoxal. DNA is then purified, click chemistry is carried out, DNA is sheared, labeled fragments are pulled down with streptavidin beads, and sequenced. (c and d) Mapping of dCas9 targets in vitro. (c) Mouse gDNA was incubated with dCas9 RNPs carrying one of two sgRNAs targeting the mouse HOXA locus. Highly specific labeling is observed at the expected target location of each sgRNA. (d) Asymmetric strand distribution of in vitro dCas9 CasKAS reads around the sgRNA target site. (e and f) Mapping of Cas9 targets in vitro. (e) Mouse gDNA was incubated with Cas9 RNPs carrying one of same two sgRNAs targeting the mouse HOXA locus. (f) The distribution of 5' read ends around targets sites in in vitro CasKAS datasets shows direct capture of the intermediate cleavage state. (g) Reproducibility of in vivo dCas9 CasKAS datasets. Shown are RPM values for 500bp windows centered on the top $\sim 7,000$ predicted target sites for the "sgRNA \#1" in two in vitro CasKAS experiments. Off-target sites are color-coded by the number of mismatches relative to the sgRNA. (h) CasKAS requires a moderate sequencing depth of $10-20 \times 10^{6}$ reads to accurately rank potential off-targets. (i-k) In vitro CasKAS maps Cas9 and dCas9 target sites. (i) Shown are CasKAS experiments with Cas9 and dCas9 and with the EMX1 sgRNA or with no sgRNA (negative control) (j) Assymmetric 5' end distribution around target sites in dCas9 in vivo CasKAS. (k) In in vivo Cas9 CasKAS, a mixture distribution is observed between phased cleavage sites and broader ssDNA labeling. 
HEK293 cells, but the dCas9/Cas9 CasKAS signal is still readily identifiable as an addition to the endogenous ssDNA enrichment pattern (Supplementary Fig. 4). These results demonstrate the utility of CasKAS for profiling CRISPR specificity both in vitro and in vivo

We next examined the genome-wide specificity of sgRNAs as measured by CasKAS. We focus on the mouse sgRNA \#1 as it displayed a substantial number of offtargets yet that number was also sufficiently small for all of them to be examined directly. We first called peaks de novo (see Methods for details) without relying on off-target prediction algorithms, then manually curated the resulting peak set, excluding peaks not exhibiting the canonical asymmetric read distribution around a fixed point on the two strands (Fig. 2a). Remarkably, while we found 32 peaks at predicted off-target sites, we also found 198 (i.e. $\sim 6 \times$ as many) additional manually curated peaks; while these peaks exhibit generally lower CasKAS signal (Fig. 2b), they all display proper peak shape characteristics (see Supplementary Fig. 13 for details), suggesting that they are genuine sites of occupancy. Most of the predicted (in total $\sim 7,500$ ) off-target sites for this sgRNA did not show substantial occupancy by dCas9 CasKAS (Fig. 2p-d).

Sequence comparison of the occupied predicted offtarget sites allowed us to evaluate determinants of Cas9 specificity (Fig. 2e). Consistent with previous reports ${ }^{2425}$, the PAM-distal region was much less sequence-constrained than the PAM-proximal seed region. We observed a similar pattern with the other sgRNAs we profiled, in both mouse and human (Supplementary Fig. 50,8 and Supplementary Fig. 912 .

When analyzing peaks not associated with predicted offtarget sites (Supplementary Fig. 14) we observed other telling patterns - at numerous sites with strong dCas9 CasKAS signal, we observe a large number of mismatches to the sgRNA sequence as well as "bulge" regions wherein indels are observed in the target sequence. These mismatches and bulges were in general much larger than what is considered permissible by off-target prediction algorithms; we speculate that the lack of consideration of potential target sequences with large numbers of mismatches or substantial insertions could explain the much larger number of such sites compared to the set of occupied predicted off-targets.

We next devised a simple metric for evaluating the degree of read clustering at cut sites (a " $C$-score"; see Methods for details) to estimate the degree of cutting by Cas9. The on-target site exhibits the second highest dCas9 CasKAS signal genome-wide. However, strikingly, even though all CasKAS-identified off-target sites showed Cas9 binding, only the on-target site displayed strong cutting activity (Fig. 2f). The behavior of other sgRNAs varies (Supplementary Fig. 5,8 and 15), with some showing multiple clearly identifiable cut sites. Overall, these results are consistent with previous reports that Cas9 requires more successful RNA:DNA basepairing for cleavage activity than is necessary for binding 26127 . Thus, interpreting the read distributions of Cas9 CasKAS at target sites enables simultaneous detection of binding specificity and the promiscuity of catalytic activity.

Finally, we compared in vitro and in vivo CasKAS profiles (Fig. $2 \mathrm{~s}$-h). We find many fewer strongly enriched sites in in vivo datasets than in vitro, with the on-target site being either the top (for dCas9) or among the top (for Cas9) sites in vivo. A potential explanation for this difference is the previously reported impediment of Cas9/dCas9 binding to DNA by the presence of nucleosomes 28 .

In conclusion, we have presented CasKAS, a simple and robust method for mapping the specificity of active and catalytically dead versions of CRISPR enzymes. CasKAS has numerous advantages over existing tools while also opening up new possibilities for studying CRISPR biology. CasKAS requires no specialized molecular biology protocols, takes just a few hours in vitro (and a similar amount of time after harvesting cells in vivo), and, due to the strong, active enrichment of target sequences, is inexpensive. In contrast to previously developed methods, It measures strand invasion by CRISPR, which is biochemically more specific and relevant to CRISPR function than DNA association. We compared de novo called CasKAS peaks to those generated by other means, and while we found large sets of peaks unique to each method, those found only by CasKAS contained much higher fractions of predicted off-target sites than those unique to other methods (Supplementary Fig. 16.

CasKAS does not rely on measuring DNA cleavage or modification and can thus be used to profile the specificity of all types of DNA-targeting CRISPR proteins. CasKAS also does not rely on cellular repair processes, cell division, or delivery of additional exogenous DNA (as in GUIDE-seq) to generate a detectable signal. These advantages, coupled with low cell input requirements, may increase the utility of the method in rare primary cell types, tissues from animal models, or even for direct assessment of specificity in edited patient cells (e.g. ex vivo edited immune cells). A current limitation of CasKAS is the requirement that a $\mathrm{G}$ nucleotide is present within the sgRNA sequence, since kethoxal requires an exposed $\mathrm{G}$ to react with. However, only a small fraction $(\leq 5 \%)$ of sgRNAs in the human genome lack any Gs for S. pyogenes PAM sequences (Supplementary Fig. 17). Another minor limitation of the current in vitro protocol is that labeling is carried out on high molecular weight (HMW) DNA and samples must be sheared serially. We have explored using pre-sheared and end-repaired DNA (to minimize kethoxal labeling of Gs on sticky ends generated by sonication), with comparable results to using HMW DNA (Supplementary Fig. 18); we anticipate that further optimization or using other approaches, such as enzymatic fragmentation, should allow the parallel high-throughput plate-based profiling of the specificity of very large numbers of sgRNAs.

In addition to being highly valuable for off-target profiling in vitro and in previously difficult to assay settings 
a
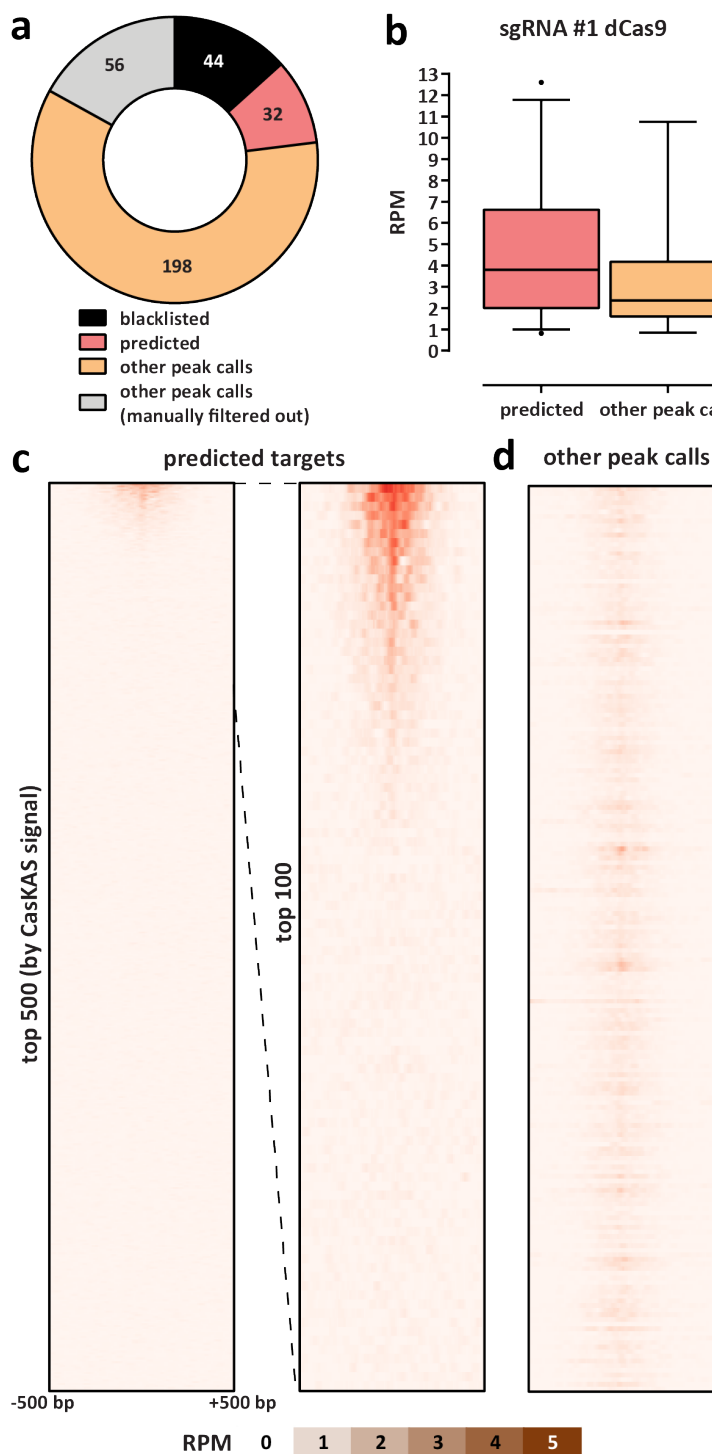

e

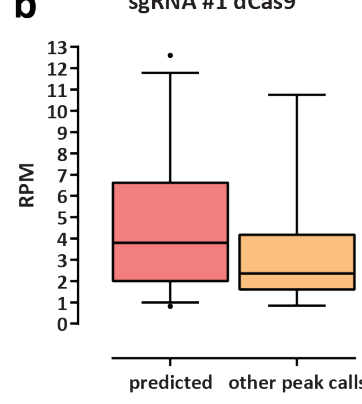

d other peak calls
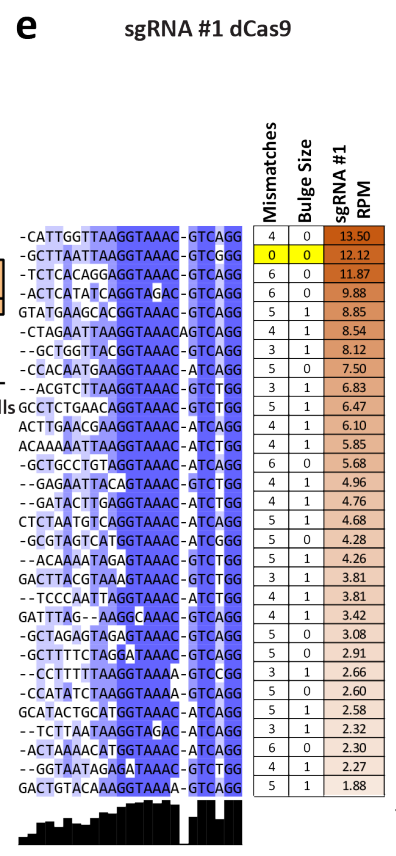

g

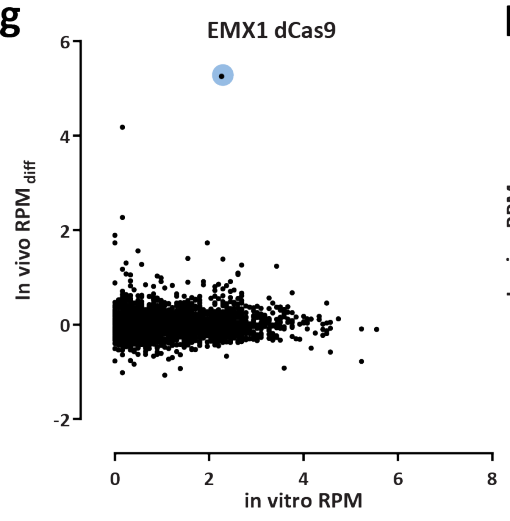

f $\quad$ sgRnA \#1 Cas9
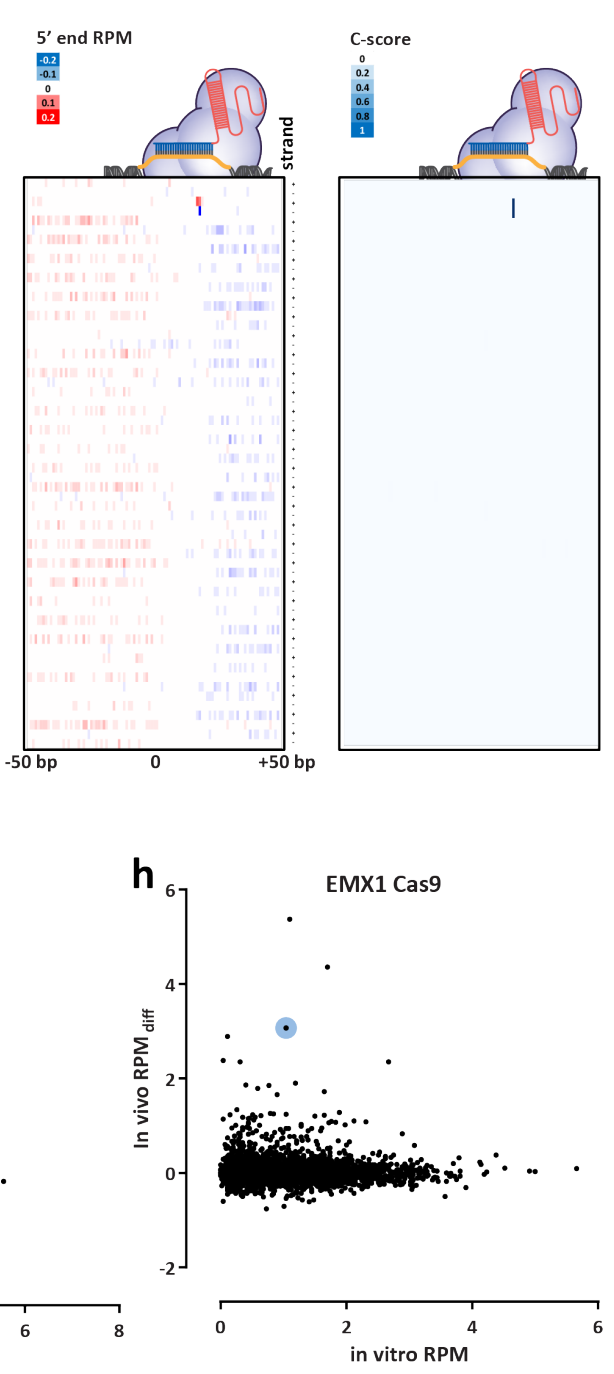

Figure 2: CasKAS profiles sgRNA specificity genome-wide. (a) Summary of de novo peak calls for sgRNA \#1 (using MACS2) (b) CasKAS signal is stronger over predicted off-target sites, but legitimate interactions are also found elsewhere in the genome. (c) CasKAS profile over predicted (by Cas-OFFinder) off-target sites for sgRNA \#1 with dCas9 (all such sites and focusing only on the top 100 ranked by dCas9 CasKAS signal). (d) CasKAS profile over peak calls outside predicted (by Cas-OFFinder) off-target sites for sgRNA \#1 with dCas9. (e) Determinants of sequence specificity as measured by dCas9 CasKAS (for sgRNA \#1). PAM-distal regions of the sgRNA are less constrained than its PAM-proximal parts. The on-target sgRNA is highlighted in yellow. (f) Active Cas9 signal read profiles can be used to distinguish off-targets associated with cutting from those where only binding occurs. Shown are the same off-target sites as in (e) and the plus- and minus-strand active Cas9 5' end profiles around the sgRNA. In this case ( $\operatorname{sgRNA} \# 1$ ), only the on-target site shows a Cas9 CasKAS pattern indicating cleavage; at the other sites even active Cas9 likely only binds but does not cut. A simple cutting score metric (" $C$-score") based on multiplying the 5 ' end forward- and reverse-strand profiles can be used to quantify cutting vs. binding. ( $\mathrm{g}$ and $\mathrm{h}$ ) Comparison between in vitro and in vivo CasKAS signal over predicted off-target sites for the EMX1 sgRNA. In vivo CasKAS is quantified as the difference in read per million ( $\pm 500 \mathrm{bp}$ of the sgRNA site) between the sgRNA KAS-seq and the no-guide control KAS-seq ("RPM $\mathrm{diff}_{\text {) }}$. The on-target site is shown in blue.

such as primary cells, we expect CasKAS to provide fruitful insights into the mechanisms and dynamics of in vivo CRISPR action (taking advantage of finely controllable
CRISPR systems such as vfCRISPR ${ }^{29}$ ), and the influence of transcriptional, regulatory, and epigenetic and other functional genomic contexts on CRISPR activity. 


\section{References}

1. Wang T, Wei JJ, Sabatini DM, Lander ES. 2014. Genetic screens in human cells using the CRISPR-Cas9 system. Science 343(6166):80-84.

2. Kim D, Bae S, Park J, Kim E, Kim S, Yu HR, Hwang J, Kim JI, Kim JS. 2015. Digenome-seq: genome-wide profiling of CRISPR-Cas9 off-target effects in human cells. Nat Methods 12(3):237-243

3. Crosetto N, Mitra A, Silva MJ, Bienko M, Dojer N, Wang Q, Karaca E, Chiarle R, Skrzypczak M, Ginalski K, Pasero P, Rowicka M, Dikic I. 2013. Nucleotideresolution DNA double-strand break mapping by nextgeneration sequencing. Nat Methods 10(4):361-365.

4. Tsai SQ, Zheng Z, Nguyen NT, Liebers M, Topkar VV, Thapar V, Wyvekens N, Khayter C, Iafrate AJ, Le LP, Aryee MJ, Joung JK. 2015. GUIDE-seq enables genome-wide profiling of off-target cleavage by CRISPR-Cas nucleases. Nat Biotechnol 33(2):187197.

5. Frock RL, Hu J, Meyers RM, Ho YJ, Kii E, Alt FW. 2015. Genome-wide detection of DNA double-stranded breaks induced by engineered nucleases. Nat Biotechnol 33(2):179-186.

6. Lensing SV, Marsico G, Hänsel-Hertsch R, Lam EY, Tannahill D, Balasubramanian S. 2016. DSBCapture: in situ capture and sequencing of DNA breaks. Nat Methods 13(10):855-857.

7. Yan WX, Mirzazadeh R, Garnerone S, Scott D, Schneider MW, Kallas T, Custodio J, Wernersson E, Li Y, Gao L, Federova Y, Zetsche B, Zhang F, Bienko M, Crosetto N. 2017. BLISS is a versatile and quantitative method for genome-wide profiling of DNA double-strand breaks. Nat Commun 8:15058.

8. Cameron P, Fuller CK, Donohoue PD, Jones BN, Thompson MS, Carter MM, Gradia S, Vidal B, Garner E, Slorach EM, Lau E, Banh LM, Lied AM, Edwards LS, Settle AH, Capurso D, Llaca V, Deschamps S, Cigan M, Young JK, May AP. 2017. Mapping the genomic landscape of CRISPR-Cas9 cleavage. Nat Methods 14(6):600-606.

9. Tsai SQ, Nguyen NT, Malagon-Lopez J, Topkar VV, Aryee MJ, Joung JK. 2017. CIRCLE-seq: a highly sensitive in vitro screen for genome-wide CRISPRCas9 nuclease off-targets. Nat Methods 14(6):607-614.

10. Schmid-Burgk JL, Gao L, Li D, Gardner Z, Strecker J, Lash B, Zhang F. 2020. Highly Parallel Profiling of Cas9 Variant Specificity. Mol Cell 78(4):794-800.e8.

11. Dobbs FM, van Eijk P, Fellows MD, Loiacono L, Nitsch R, Reed SH. 2020. Precision digital mapping of endogenous and induced genomic DNA breaks by INDUCE-seq. bioRxiv 2020.08.25.266239

12. Lazzarotto CR, Malinin NL, Li Y, Zhang R, Yang Y, Lee G, Cowley E, He Y, Lan X, Jividen K, Katta V, Kolmakova NG, Petersen CT, Qi Q, Strelcov E, Maragh S, Krenciute G, Ma J, Cheng Y, Tsai SQ. 2020. CHANGE-seq reveals genetic and epigenetic effects on CRISPR-Cas9 genome-wide activity. Nat Biotechnol 38(11):1317-1327.

13. Wienert B, Wyman SK, Richardson CD, Yeh CD, Akcakaya P, Porritt MJ, Morlock M, Vu JT, Kazane KR, Watry HL, Judge LM, Conklin BR, Maresca M, Corn JE. 2019. Unbiased detection of CRISPR off-targets in vivo using DISCOVER-Seq. Science 364(6437):286289.

14. Wu X, Scott DA, Kriz AJ, Chiu AC, Hsu PD, Dadon DB, Cheng AW, Trevino AE, Konermann S, Chen S, Jaenisch R, Zhang F, Sharp PA. 2014. Genome-wide binding of the CRISPR endonuclease Cas9 in mammalian cells. Nat Biotechnol 32(7):670-676.

15. Kuscu C, Arslan S, Singh R, Thorpe J, Adli M. 2014. Genome-wide analysis reveals characteristics of offtarget sites bound by the Cas9 endonuclease. Nat Biotechnol 32(7):677-683.

16. Höijer I, Johansson J, Gudmundsson S, Chin CS, Bunikis I, Häggqvist S, Emmanouilidou A, Wilbe M, den Hoed M, Bondeson ML, Feuk L, Gyllensten U, Ameur A. 2020. Amplification-free long-read sequencing reveals unforeseen CRISPR-Cas9 off-target activity. Genome Biol 21(1):290.

17. Doench JG, Fusi N, Sullender M, Hegde M, Vaimberg EW, Donovan KF, Smith I, Tothova Z, Wilen C, Orchard R, Virgin HW, Listgarten J, Root DE. 2016. Optimized sgRNA design to maximize activity and minimize off-target effects of CRISPR-Cas9. Nat Biotechnol 34(2):184-191.

18. Perez AR, Pritykin Y, Vidigal JA, Chhangawala S, Zamparo L, Leslie CS, Ventura A. 2017. GuideScan software for improved single and paired CRISPR guide RNA design. Nat Biotechnol 35(4):347-349.

19. Wu T, Lyu R, You Q, He C. 2020. Kethoxal-assisted single-stranded DNA sequencing captures global transcription dynamics and enhancer activity in situ. Nat Methods 17(5):515-523.

20. Landt SG, Marinov GK, Kundaje A, Kheradpour P, Pauli F, Batzoglou S, Bernstein BE, Bickel P, Brown JB, Cayting P, Chen Y, DeSalvo G, Epstein C, Fisher-Aylor KI, Euskirchen G, Gerstein M, Gertz J, Hartemink AJ, Hoffman MM, Iyer VR, Jung YL, Karmakar S, Kellis M, Kharchenko PV, Li Q, Liu T, Liu XS, Ma L, Milosavljevic A, Myers RM, Park PJ, Pazin MJ, Perry MD, Raha D, Reddy TE, Rozowsky J, Shoresh N, Sidow A, Slattery M, Stamatoyannopoulos JA, Tolstorukov MY, White KP, Xi S, Farnham PJ, Lieb JD, Wold BJ, Snyder M. 2012. ChIP-seq guidelines and practices of the ENCODE and modENCODE consortia. Genome Res 22(9):1813-1831.

21. Richardson CD, Ray GJ, DeWitt MA, Curie GL, Corn JE. 2016. Enhancing homology-directed genome editing by catalytically active and inactive CRISPRCas9 using asymmetric donor DNA. Nat Biotechnol 34(3):339-344.

22. Gisler S, Gonalves JP, Akhtar W, de Jong J, Pindyurin 
AV, Wessels LFA, van Lohuizen M. 2019. Multiplexed Cas9 targeting reveals genomic location effects and gRNA-based staggered breaks influencing mutation efficiency. Nat Commun 10(1):1598.

23. Jones SK Jr, Hawkins JA, Johnson NV, Jung C, Hu K, Rybarski JR, Chen JS, Doudna JA, Press WH, Finkelstein IJ. 2021. Massively parallel kinetic profiling of natural and engineered CRISPR nucleases. Nat Biotechnol 39(1):84-93.

24. Hsu PD, Scott DA, Weinstein JA, Ran FA, Konermann S, Agarwala V, Li Y, Fine EJ, Wu X, Shalem O, Cradick TJ, Marraffini LA, Bao G, Zhang F. 2013. DNA targeting specificity of RNA-guided Cas9 nucleases. Nat Biotechnol 31(9):827-832.

25. Semenova E, Jore MM, Datsenko KA, Semenova A, Westra ER, Wanner B, van der Oost J, Brouns SJ, Severinov K. 2011. Interference by clustered regularly interspaced short palindromic repeat (CRISPR) RNA is governed by a seed sequence. Proc Natl Acad Sci U $S$ A 108(25):10098-10103.

26. Dahlman JE, Abudayyeh OO, Joung J, Gootenberg JS, Zhang F, Konermann S. 2015. Orthogonal gene knockout and activation with a catalytically active Cas9 nuclease. Nat Biotechnol 33(11):1159-1161.

27. Kiani S, Chavez A, Tuttle M, Hall RN, Chari R, TerOvanesyan D, Qian J, Pruitt BW, Beal J, Vora S, Buchthal J, Kowal EJ, Ebrahimkhani MR, Collins JJ, Weiss R, Church G. 2015. Cas9 gRNA engineering for genome editing, activation and repression. Nat Methods 12(11):1051-1054
28. Horlbeck MA, Witkowsky LB, Guglielmi B, Replogle JM, Gilbert LA, Villalta JE, Torigoe SE, Tjian R, Weissman JS. 2016. Nucleosomes impede Cas9 access to DNA in vivo and in vitro. Elife 5:e12677.

29. Liu Y, Zou RS, He S, Nihongaki Y, Li X, Razavi S, Wu B, Ha T. 2020. Very fast CRISPR on demand. Science 368(6496):1265-1269.

30. Langmead B, Trapnell C, Pop M, Salzberg SL. 2009. Ultrafast and memory-efficient alignment of short DNA sequences to the human genome. Genome Biol 10(3):R25.

31. Feng J, Liu T, Qin B, Zhang Y, Liu XS. 2012. Identifying ChIP-seq enrichment using MACS. Nat Protoc 7(9):1728-1740.

32. Amemiya HM, Kundaje A, Boyle AP. 2019. The ENCODE Blacklist: Identification of Problematic Regions of the Genome. Sci Rep 9(1):9354.

33. Bae S, Park J, Kim JS. 2014. Cas-OFFinder: a fast and versatile algorithm that searches for potential offtarget sites of Cas9 RNA-guided endonucleases. Bioinformatics 30(10):1473-1475.

34. Edgar RC. 2004. MUSCLE: multiple sequence alignment with high accuracy and high throughput. Nucleic Acids Res 32(5):1792-1797.

35. Waterhouse AM, Procter JB, Martin DM, Clamp M, Barton GJ. 2009. Jalview Version 2-a multiple sequence alignment editor and analysis workbench. Bioinformatics 25(9):1189-1191. 


\section{Methods}

\section{Guide RNA sequences}

Guide RNAs were obtained from IDT ("sgRNA \#1" and "sgRNA \#2") or from Synthego (all others).

The following sgRNA sequences were used in this study:

1. "sgRNA \#1": GCTTAATTAagGTAaACGTC

2. "sgRNA \#2": CCAACCTGGCGGCTCGTTGG

3. "EMX1_Tsai": GAGTCCGAGCAGAAGAAGAA

4. "VEGFA-site1": GGGTGGGGGGAGTTTGCTCC

5. "Nanog-sg2": GATCTCTAGTGGGAAGTTTC

6. "Nanog-sg3": GTCTGTAGAAAGAATGGAAG

Guide RNAs were dissolved to a concentration of 100 $\mu \mathrm{M}$ using nuclease-free $1 \times \mathrm{TE}$ buffer and stored at $-20^{\circ} \mathrm{C}$.

\section{In vitro CasKAS}

In vitro CasKAS experiments were executed as follows.

First, $1 \mu \mathrm{L}$ of each synthetic sgRNA were incubated at room temperature with $1 \mu \mathrm{L}$ of recombinant purified dCas9 (MCLab dCAS9B-200) for 20 minutes. The RNP was then incubated with $1 \mu \mathrm{g}$ of gDNA at $37^{\circ} \mathrm{C}$ for 10 minutes.

The KAS reaction was then carried out by adding 1 $\mu \mathrm{L}$ of $500 \mathrm{mM} \mathrm{N}_{3}$-kethoxal (ApeXBio A8793). DNA was immediately purified using the MinElute PCR Purification Kit (Qiagen 28006), and eluted in 87.5 or $175 \mu \mathrm{L} 25 \mathrm{mM}$ $\mathrm{K}_{3} \mathrm{BO}_{3}$.

\section{In vivo CasKAS}

For in vivo CasKAS experiments, HEK293T cells were seeded at 400,000 cells/well into a 6 -well plate the day before RNP transfection. Media was exchanged 2 hours before transfection. For each well, 6,250 ng of Cas9 (MCLAB CAS9-200) or dCas9 (MCLAB dCAS9B-200) and 1,200 ng sgRNA was complexed with CRISPRMAX (Thermo Fisher CMAX00008) reagent in Opti-MEM (Thermo Fisher 51985091) following manufacturer's protocol. After incubation at room temperature for 15 minutes, the RNP solution was directly added to each well and gently mixed. The cells were incubated with the RNP complex for 14 hours at $37^{\circ} \mathrm{C}$. To harvest and perform kethoxal labeling, media was removed and room temperature $1 \times$ PBS was used to wash the cells. Cells were then dissociated with trypsin, trypsin was quenched with media, cells were pelleted at room temperature, and then resuspended in $100 \mu \mathrm{L}$ of media supplemented with $5 \mathrm{M} \mathrm{N}_{3}$-kethoxal (final concentration). Cells were incubated for 10 minutes at $37^{\circ} \mathrm{C}$ with shaking at 500 rpm in a Thermomixer. Cells were then pelleted by centrifuging at $500 \mathrm{~g}$ for 5 minutes at $4{ }^{\circ} \mathrm{C}$. Genomic DNA was then extracted using the Monarch gDNA Purification Kit (NEB T3010S) following the standard protocol but with elution using $175 \mu \mathrm{L} 25 \mathrm{mM} \mathrm{K}_{3} \mathrm{BO}_{3}$ at $\mathrm{pH}$ 7.0.

\section{Click reaction, biotin pull down and library generation}

The click reaction was carried out by combining $175 \mu \mathrm{L}$ purified DNA, $5 \mu \mathrm{L} 20 \mathrm{mM}$ DBCO-PEG4-biotin (DMSO solution, Sigma 760749), and $20 \mu \mathrm{L} 10 \times$ PBS in a final volume of $200 \mu \mathrm{L}$ or $87.5 \mu \mathrm{L}$ purified and sheared DNA, $2.5 \mu \mathrm{L} 20$ mM DBCO-PEG4-biotin (DMSO solution, Sigma 760749), and $10 \mu \mathrm{L} 10 \times$ PBS in a final volume of $100 \mu \mathrm{L}$. The reaction was incubated at $37^{\circ} \mathrm{C}$ for 90 minutes.

DNA was purified using AMPure XP beads $(50 \mu \mathrm{L}$ for a $100 \mu \mathrm{L}$ reaction or $100 \mu \mathrm{L}$ for a $200 \mu \mathrm{L}$ reaction), beads were washed on a magnetic stand twice with $80 \% \mathrm{EtOH}$, and eluted in $130 \mu \mathrm{L} 25 \mathrm{mM} \mathrm{K}_{3} \mathrm{BO}_{3}$.

Purified DNA was then sheared on a Covaris E220 instrument down to $\sim 150-400$ bp size.

For streptavidin pulldown of biotin-labeled DNA, $10 \mu \mathrm{L}$ of $10 \mathrm{mg} / \mathrm{mL}$ Dynabeads MyOne Streptavidin T1 beads (Life Technologies, 65602) were separated on a magnetic stand, then washed with $300 \mu \mathrm{L}$ of $1 \times$ TWB (Tween Washing Buffer; $5 \mathrm{mM}$ Tris-HCl pH 7.5; $0.5 \mathrm{mM}$ EDTA; $1 \mathrm{M}$ $\mathrm{NaCl} ; 0.05 \%$ Tween 20). The beads were resuspended in $300 \mu \mathrm{L}$ of $2 \times$ Binding Buffer $(10 \mathrm{mM}$ Tris- $\mathrm{HCl} \mathrm{pH} \mathrm{7.5,1}$ mM EDTA; $2 \mathrm{M} \mathrm{NaCl}$ ), the sonicated DNA was added (diluted to a final volume of $300 \mu \mathrm{L}$ if necessary), and the beads were incubated for $\geq 15$ minutes at room temperature on a rotator. After separation on a magnetic stand, the beads were washed with $300 \mu \mathrm{L}$ of $1 \times \mathrm{TWB}$, and heated at $55^{\circ} \mathrm{C}$ in a Thermomixer with shaking for 2 minutes. After removal of the supernatant on a magnetic stand, the TWB wash and $55^{\circ} \mathrm{C}$ incubation were repeated.

Final libraries were prepared on beads using the NEBNext Ultra II DNA Library Prep Kit (NEB, \#E7645) as follows. End repair was carried out by resuspending beads in $50 \mu \mathrm{L} 1 \times \mathrm{EB}$ buffer, and adding $3 \mu \mathrm{L}$ NEB Ultra End Repair Enzyme and $7 \mu \mathrm{L}$ NEB Ultra End Repair Enzyme, followed by incubation at $20^{\circ} \mathrm{C}$ for 30 minutes (in a Thermomixer, with shaking at $1,000 \mathrm{rpm}$ ) and then at $65^{\circ} \mathrm{C}$ for 30 minutes.

Adapters were ligated to DNA fragments by adding 30 $\mu \mathrm{L}$ Blunt Ligation mix, $1 \mu \mathrm{L}$ Ligation Enhancer and $2.5 \mu \mathrm{L}$ NEB Adapter, incubating at $20^{\circ} \mathrm{C}$ for 20 minutes, adding 3 $\mu \mathrm{L}$ USER enzyme, and incubating at $37^{\circ} \mathrm{C}$ for 15 minutes (in a Thermomixer, with shaking at 1,000 rpm) .

Beads were then separated on a magnetic stand, and washed with $300 \mu \mathrm{L}$ TWB for 2 minutes at $55^{\circ} \mathrm{C}, 1000$ rpm in a Thermomixer. After separation on a magnetic stand, beads were washed in $100 \mu \mathrm{L} 0.1 \times \mathrm{TE}$ buffer, then resuspended in $15 \mu \mathrm{L} 0.1 \times \mathrm{TE}$ buffer, and heated at $98^{\circ} \mathrm{C}$ for 10 minutes.

For PCR, $5 \mu \mathrm{L}$ of each of the i5 and i7 NEB Next sequencing adapters were added together with $25 \mu \mathrm{L} 2 \times \mathrm{NEB}$ Ultra PCR Mater Mix. PCR was carried out with a $98^{\circ} \mathrm{C}$ incubation for 30 seconds and 12 cycles of $98^{\circ} \mathrm{C}$ for $10 \mathrm{sec}-$ onds, $65^{\circ} \mathrm{C}$ for 30 seconds, and $72^{\circ} \mathrm{C}$ for 1 minute, followed by incubation at $72^{\circ} \mathrm{C}$ for 5 minutes. 
Beads were separated on a magnetic stand, and the supernatant was cleaned up using $1.8 \times$ AMPure XP beads.

Libraries were sequenced in a paired-end format on an Illumina NextSeq instrument using NextSeq 500/550 high output kits $(2 \times 36$ cycles $)$.

\section{Data processing}

Demultipexed fastq files were mapped to the hg38 assembly of the human genome or the mm10 version of the mouse genome as $2 \times 36$ mers using Bowtie ${ }^{30}$ with the following settings: -v 2 -k 2 -m 1 --best --strata -X 1000. Duplicate reads were removed using picard-tools (version 1.99).

Browser tracks generation, fragment length estimation, TSS enrichment calculations, and other analyses were carried out using custom-written Python scripts (https: //github.com/georgimarinov/GeorgiScripts). The refSeq set of annotations were used for evaluation of enrichment around TSSs.

\section{Peak calling}

Peak calling on in vitro binding datasets was carried out using version 2.1.0 of MACS2 ${ }^{31}$ with default settings.

Peaks were then compared against the ENCODE set of "blacklisted" regions ${ }^{32}$ to filter out likely artifacts.

\section{Sequence analysis}

Guide RNA off-target predictions were obtained from CasOFFinder 33

Multiple sequence alignments of sgRNA sequences and their off-targets were generated using MUSCLE ${ }^{34}$ and visualized using JalView 35 .

\section{Quantification}

\section{Cutting score calculation}

The Cas9 cutting $C$-score was calculated as follows.

First, basepair-level Read-Per-Million (RPM) profiles for mapped read 5' ends were generated separately for the forward and reverse strands. Then the $C$-score was calculated by multiply the forward and reverse strand profiles (summed over a running window of $3 \mathrm{bp}$ ):

$$
C \text { - } \text { score }_{c, i}=\sum_{j=i-1}^{j=i+1} R P M_{c, j}^{+} \times \sum_{j=i-1}^{j=i+1} R P M_{c, j}^{-}
$$

Where $c, i$ indicate the coordinates by chromosome and position.

\section{Data availability}

Sequencing reads for the datasets described in this study are available from GEO accession GSE171962.

\section{Author contributions}

G.K.M. conceptualized the study, performed initial in vitro CasKAS experiments, analyzed data, and wrote the manuscript with input from all authors. S.H.K. developed the in vivo CasKAS protocol and performed in vivo CasKAS experiments. S.T.B. carried out in vitro CasKAS optimization. A.E.T. and J.T. supplied sgRNAs and designed off-target profiling experiments. A.E.T. carried out off-target analysis for mouse sgRNAs. T.W. provided key reagents. W.J.G., A.K., C.H. M.C.B. and L.B. supervised the study.

\section{Acknowledgments}

This work was supported by NIH grants (P50HG007735, RO1 HG008140, U19AI057266 and UM1HG009442 to W.J.G., 1UM1HG009436 to W.J.G. and A.K., 1DP2OD022870-01 and 1U01HG009431 to A.K., and HG006827 to C.H.), the Rita Allen Foundation (to W.J.G.), the Baxter Foundation Faculty Scholar Grant, and the Human Frontiers Science Program grant RGY006S (to W.J.G). W.J.G is a Chan Zuckerberg Biohub investigator and acknowledges grants 2017-174468 and 2018-182817 from the Chan Zuckerberg Initiative. S.K. is supported by MSTP training grant T32GM007365 and the Paul and Daisy Soros Fellowship. J.T. is supported by the NIDDK F99/K00 fellowship of the National Institutes of Health (F99DK126120). M.C.B. is supported by a grant from Stanford ChEM-H and an NIH Directors New Innovator Award (1DP2HD08406901). Fellowship support also provided by the Stanford School of Medicine Dean's Fellowship (G.K.M.), the Siebel Scholars, the Enhancing Diversity in Graduate Education Program and the Weiland Family Fellowship (A.E.T.). C.H. is a Howard Hughes Medical Institute Investigator.

The authors would like to thank Zohar Shipony and members of the Greenleaf, Kundaje, and Bassik labs for helpful discussion and suggestions regarding this work.

\section{Competing interests}

G.K.M., W.J.G, T.W. and C.H. have submitted a provisional patent application based on this work. 
bioRxiv preprint doi: https://doi.org/10.1101/2021.04.16.440202; this version posted April 17, 2021. The copyright holder for this preprint (which

was not certified by peer review) is the author/funder, who has granted bioRxiv a license to display the preprint in perpetuity. It is made available under aCC-BY-NC-ND 4.0 International license.

\section{Supplementary Materials}

\section{Supplementary Figures}

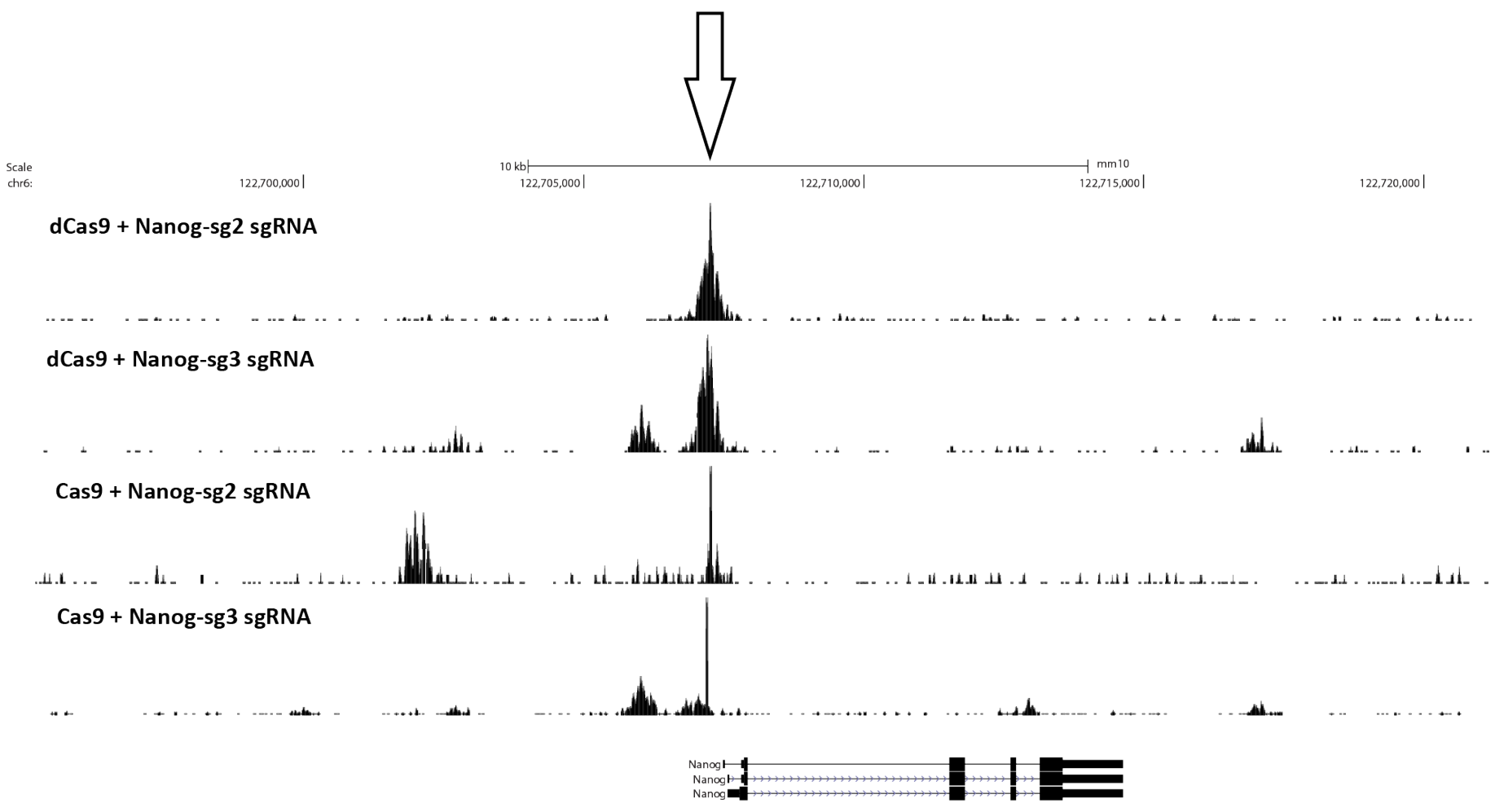

Supplementary Figure 1: In vitro dCas9 and Cas9 CasKAS profiles around the mouse Nanog locus using the "Nanog-sg2" and "Nanog-sg3" sgRNAs. 


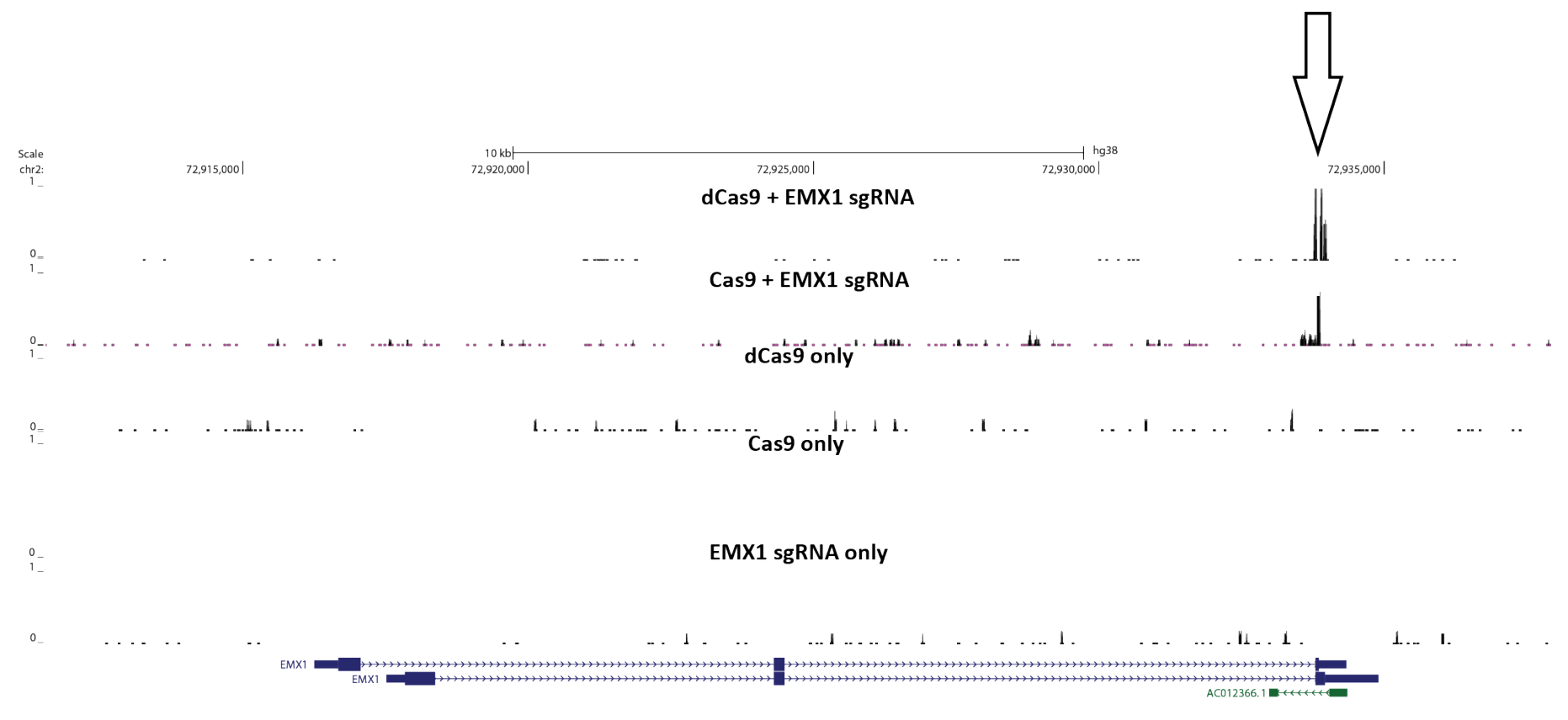

Supplementary Figure 2: CasKAS signal in vitro is specific to the activity of the dCas9/Cas9 protein combined with its sgRNA. CasKAS was carried out with the EMX1 sgRNA and with the following combinations of protein and sgRNA: dCas9 + sgRNA, Cas9 + sgRNA, dCas9 alone, Cas9 alone, or sgRNA alone.

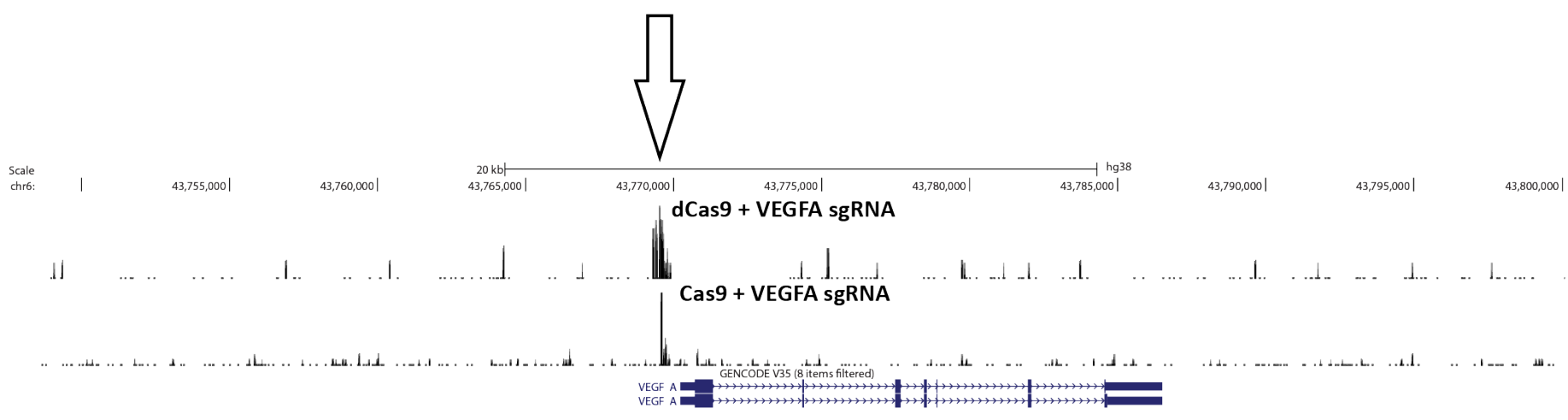

Supplementary Figure 3: CasKAS signal in vitro around the VEGFA gene with the VEGFA sgRNA. 
bioRxiv preprint doi: https://doi.org/10.1101/2021.04.16.440202; this version posted April 17, 2021. The copyright holder for this preprint (which was not certified by peer review) is the author/funder, who has granted bioRxiv a license to display the preprint in perpetuity. It is made available under aCC-BY-NC-ND 4.0 International license.

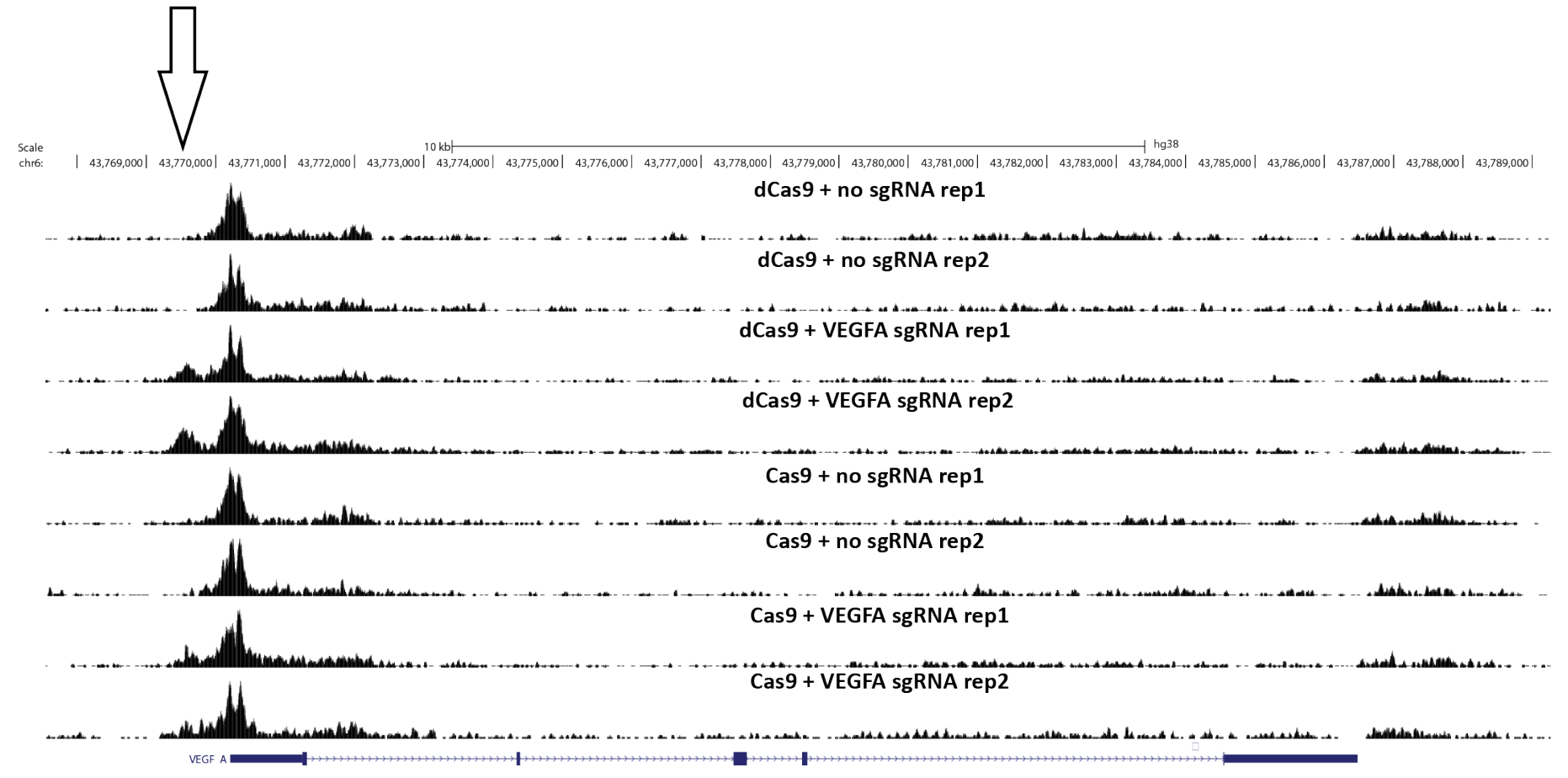

Supplementary Figure 4: CasKAS signal in vivo around the VEGFA gene with the VEGFA sgRNA. 
a

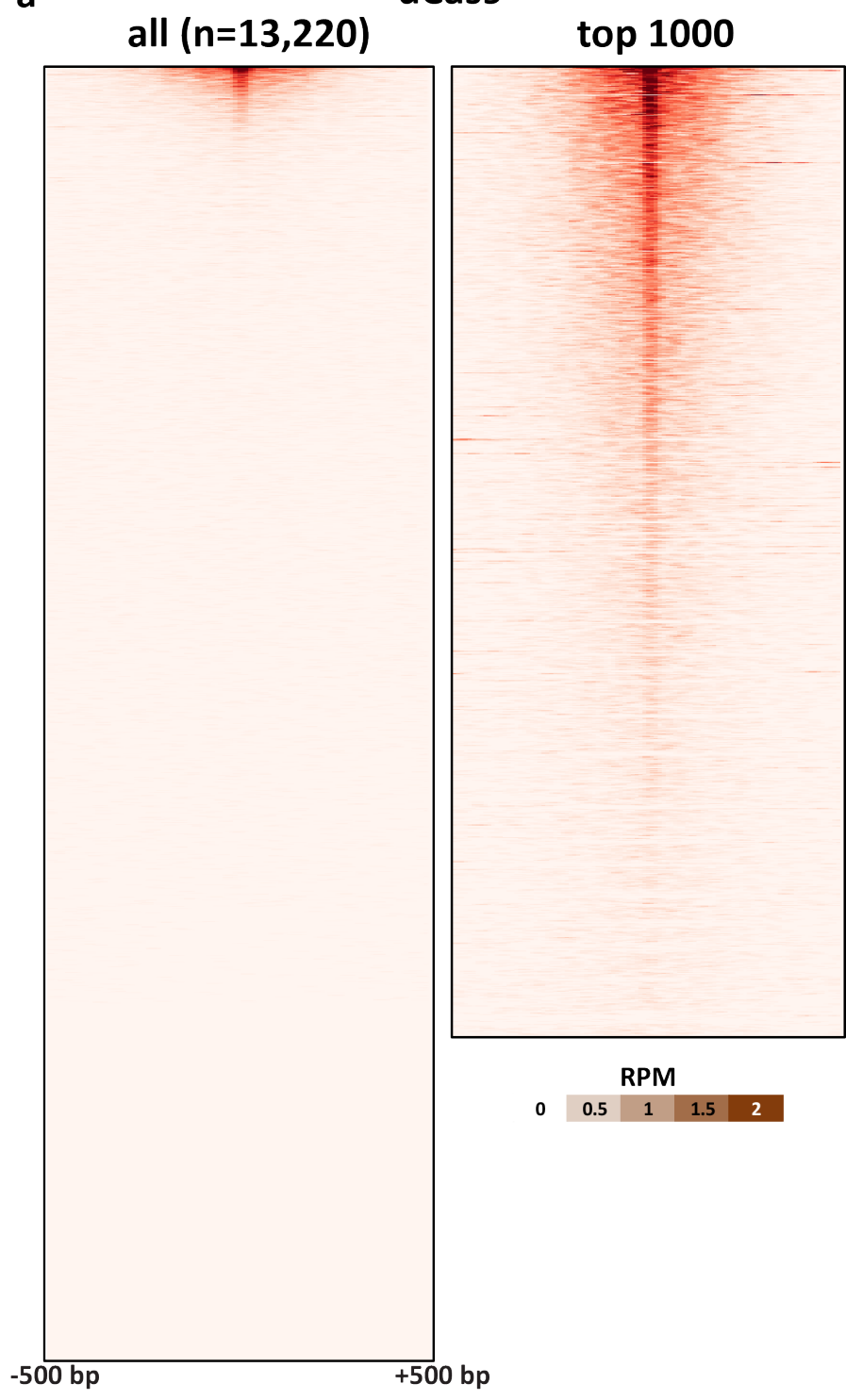

b

all $(n=13,220)$

Cas9

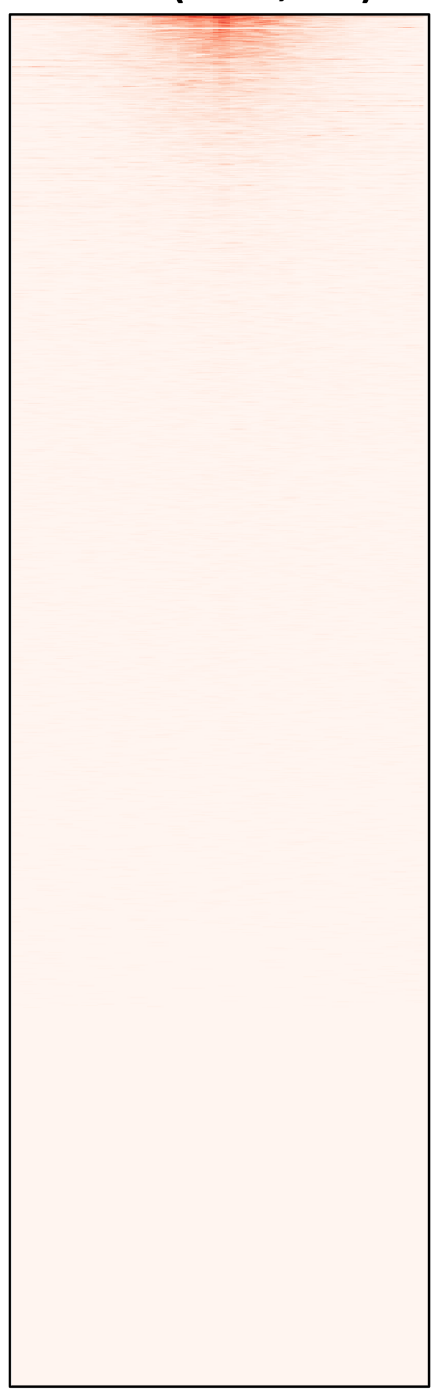

top 1000

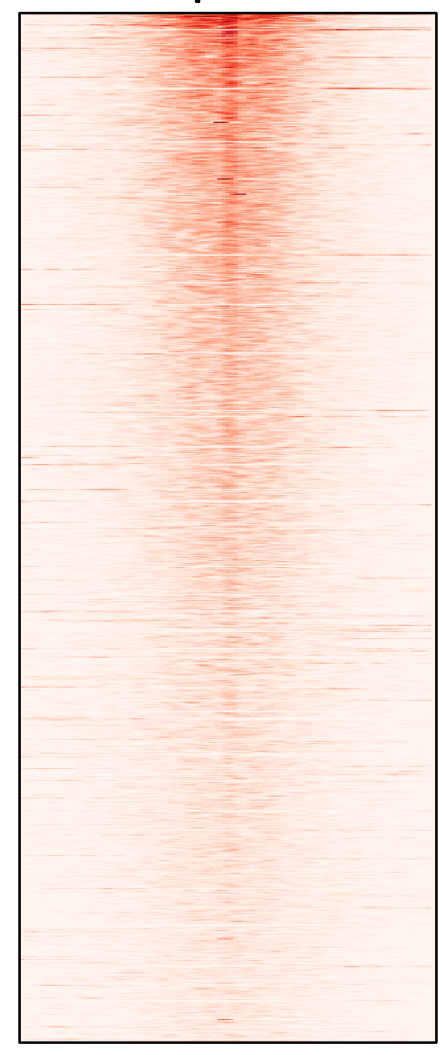

Supplementary Figure 5: In vitro dCas9 and Cas9 CasKAS profiles for the "Nanog-sg2" sgRNA. CasKAS profiles are shown for all off-target sites predicted by Cas-OFFinder as well as for the top 1000 sites (ranked by CasKAS $\mathrm{RPM}$ values over the $\pm 500 \mathrm{bp}$ region around the sgRNA target site). 
a
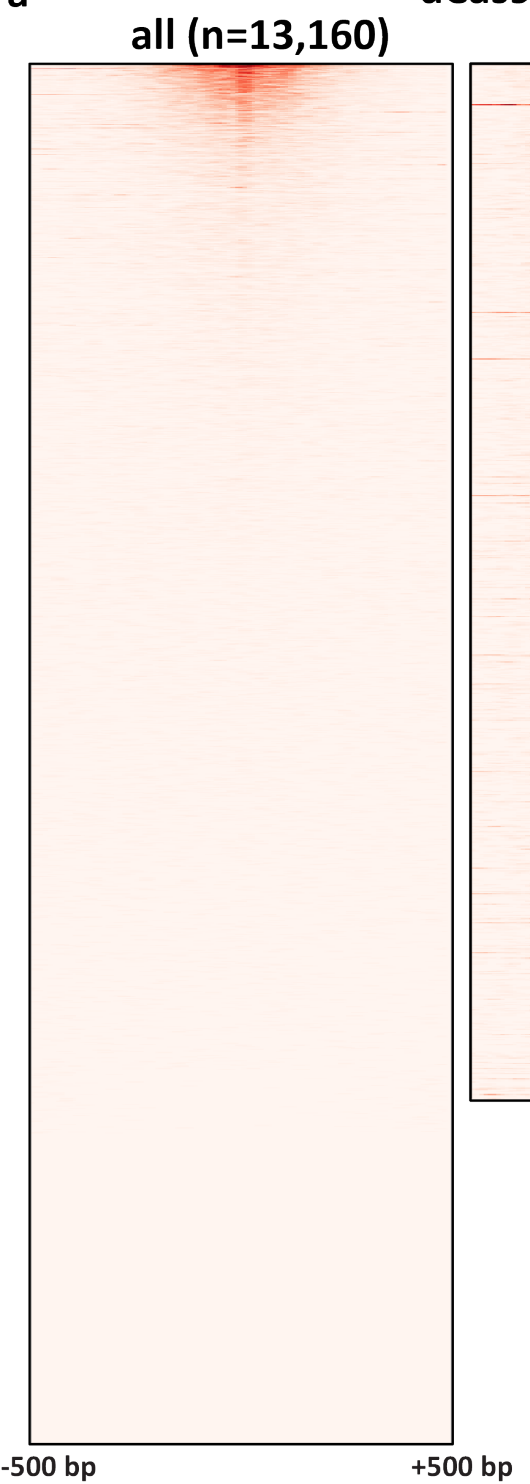

b top 1000

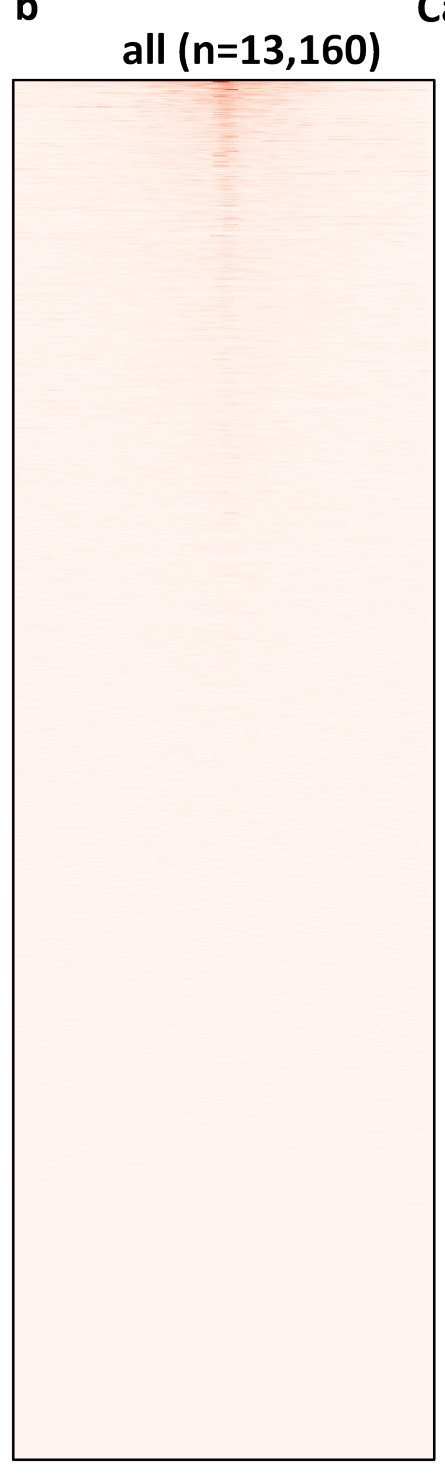

Cas9
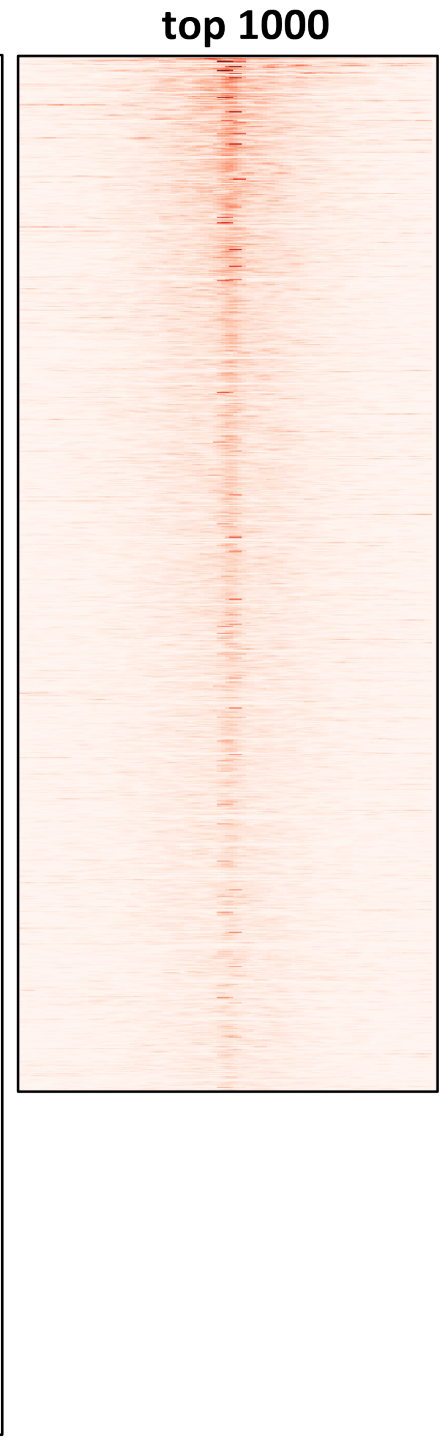

Supplementary Figure 6: In vitro dCas9 and Cas9 CasKAS profiles for the "Nanog-sg3" sgRNA. CasKAS profiles are shown for all off-target sites predicted by Cas-OFFinder as well as for the top 1000 sites (ranked by CasKAS $\mathrm{RPM}$ values over the $\pm 500 \mathrm{bp}$ region around the sgRNA target site). 
a
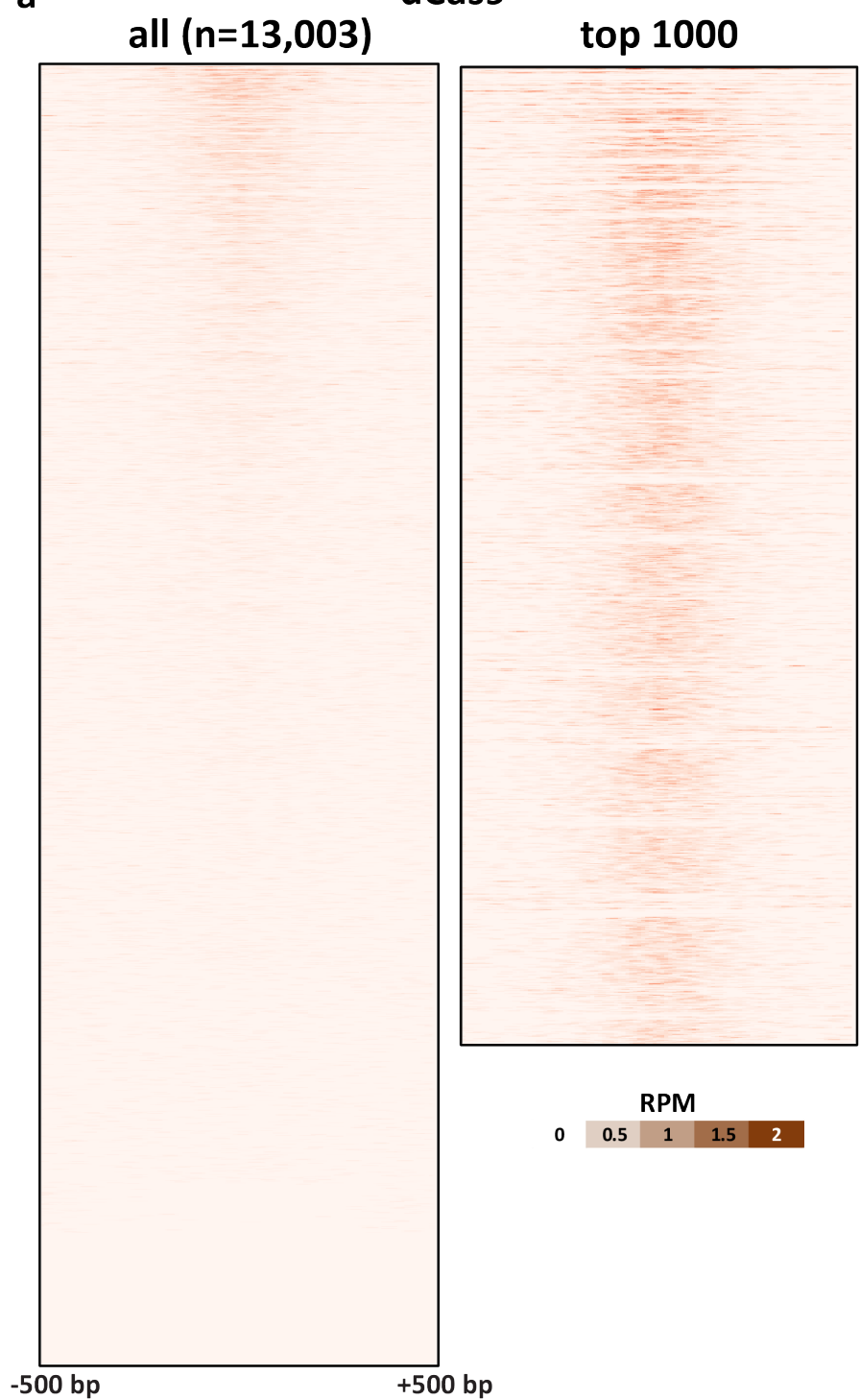

b all $(n=13,003)$

Cas9

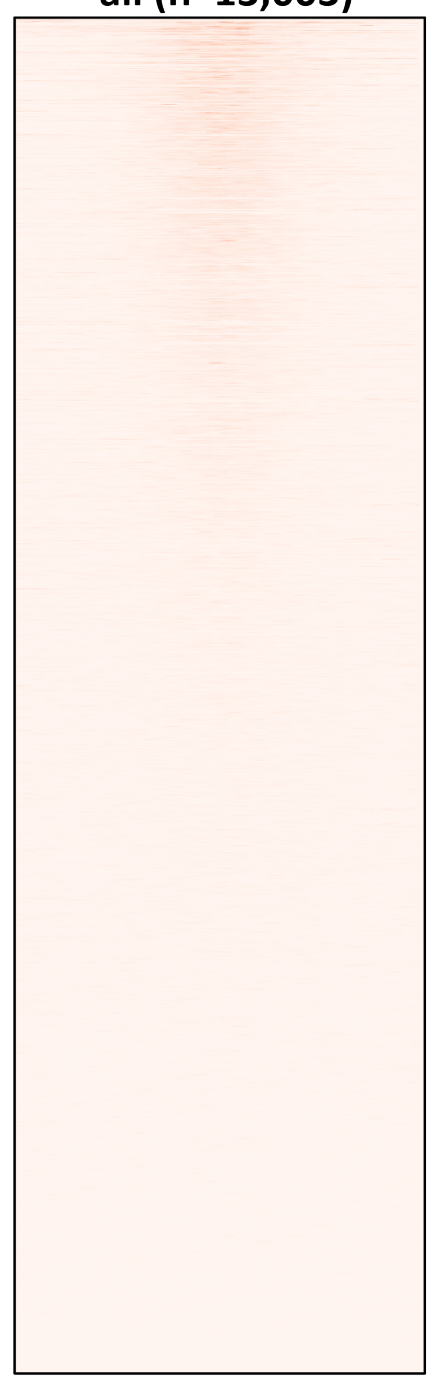

top 1000

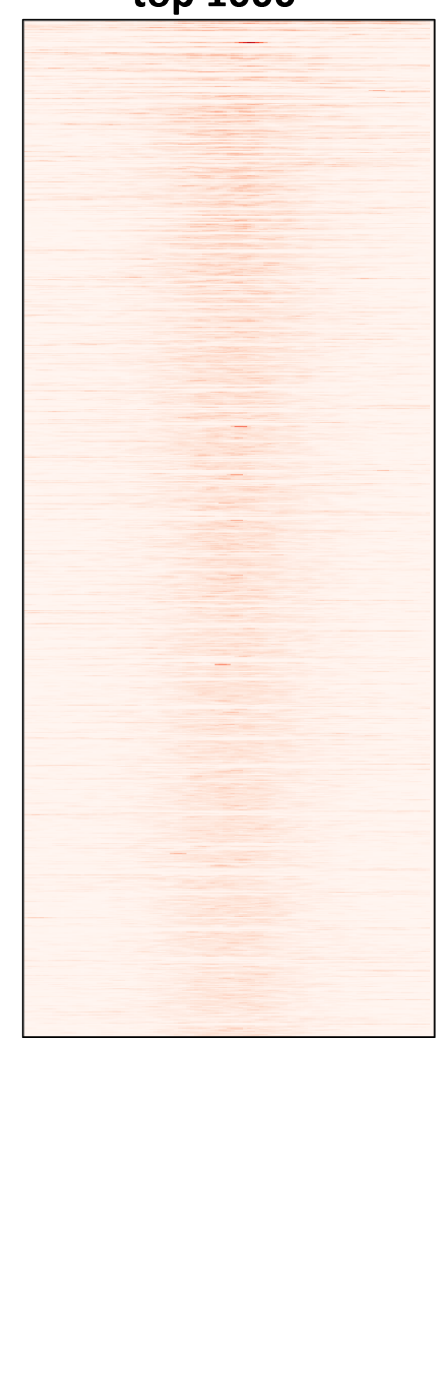

Supplementary Figure 7: In vitro dCas9 and Cas9 CasKAS profiles for the "EMX1_Tsai" sgRNA. CasKAS profiles are shown for all off-target sites predicted by Cas-OFFinder as well as for the top 1000 sites (ranked by CasKAS RPM values over the $\pm 500 \mathrm{bp}$ region around the sgRNA target site). 
a

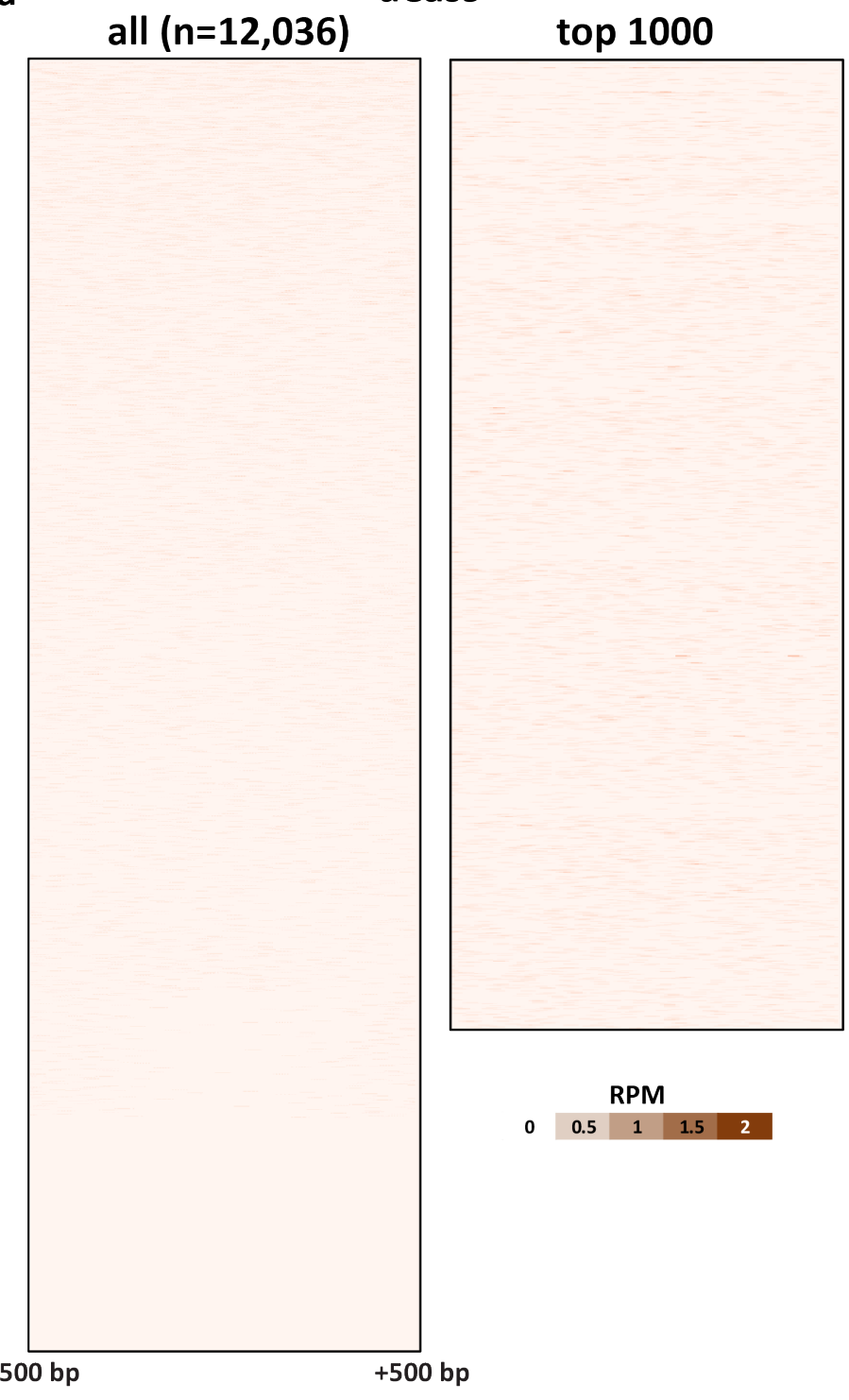

b

all $(n=12,036)$

Cas9

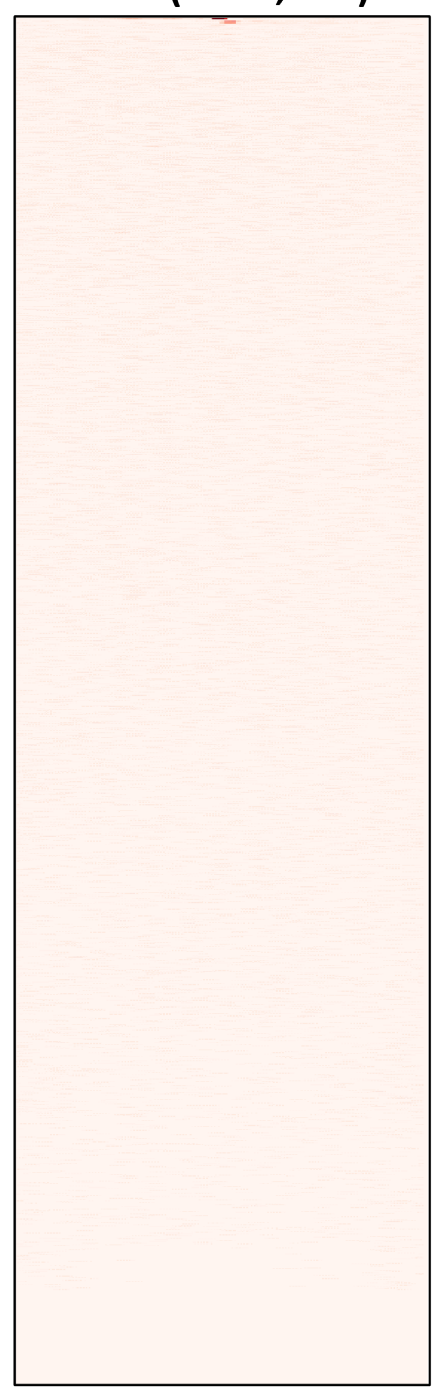

top 1000

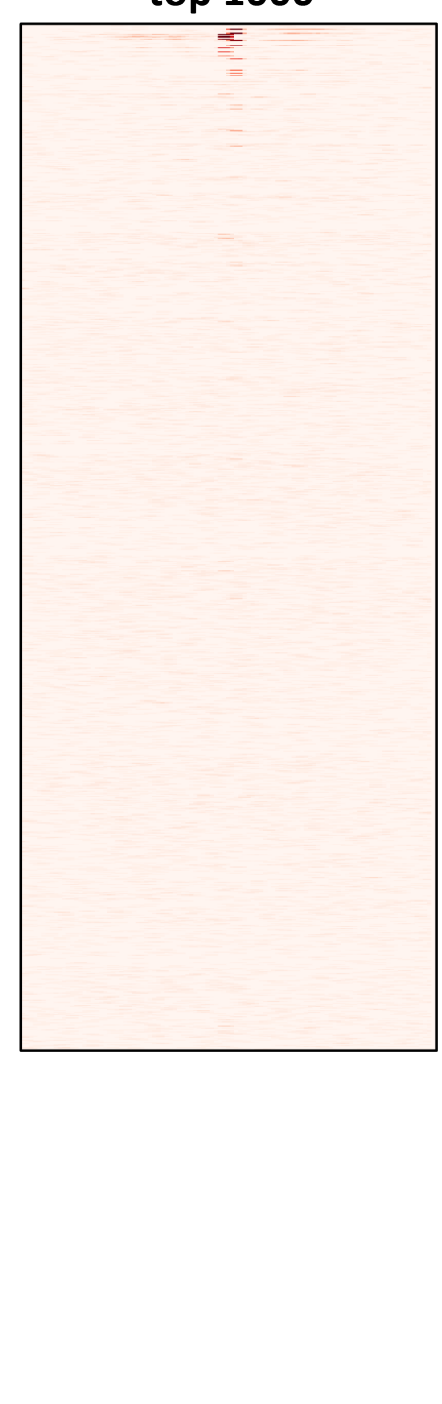

Supplementary Figure 8: In vitro dCas9 and Cas9 CasKAS profiles for the "VEGFA-site1" sgRNA. CasKAS profiles are shown for all off-target sites predicted by Cas-OFFinder as well as for the top 1000 sites (ranked by CasKAS $\mathrm{RPM}$ values over the $\pm 500 \mathrm{bp}$ region around the sgRNA target site). 
bioRxiv preprint doi: https://doi.org/10.1101/2021.04.16.440202; this version posted April 17, 2021. The copyright holder for this preprint (which was not certified by peer review) is the author/funder, who has granted bioRxiv a license to display the preprint in perpetuity. It is made available under aCC-BY-NC-ND 4.0 International license.

a dCas9

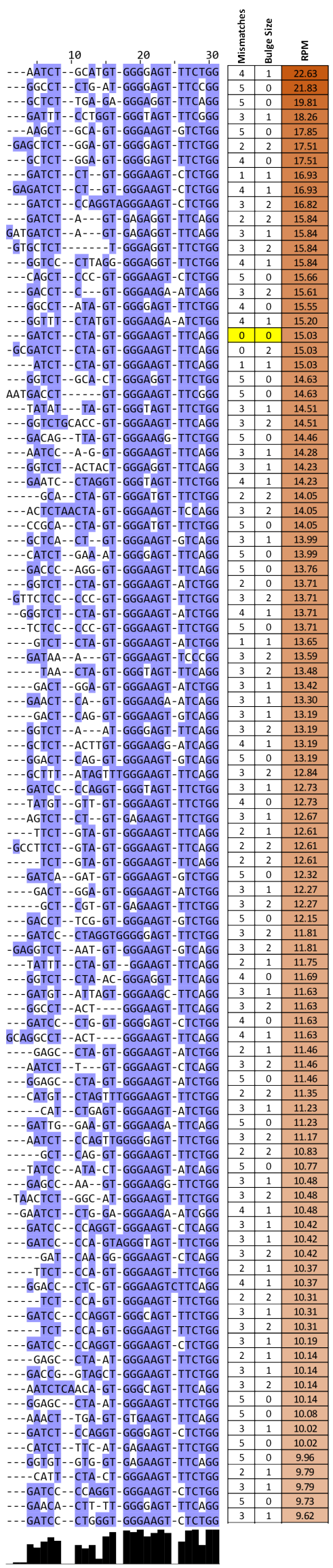

b

\section{Cas9}

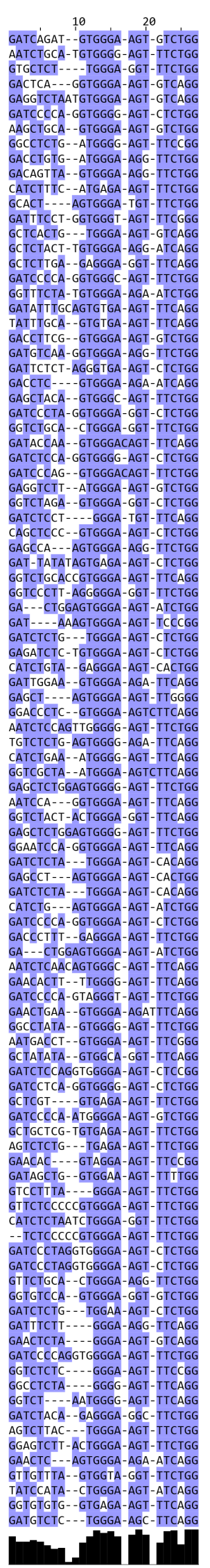

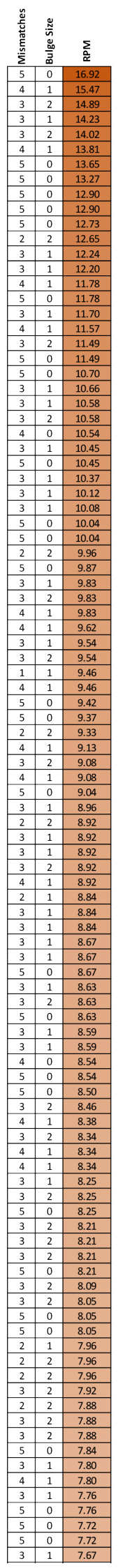

Supplementary Figure 9: Multiple sequence alignment of offtarget sites identified by in vitro dCas9 and Cas9 CasKAS for the "Nanog-sg2" sgRNA. Shown are the top 100 off-target sites as predicted by Cas-OFFinder and ranked by CasKAS signal. The on-target site (if within the top 100) is highlighted in yellow. 
bioRxiv preprint doi: https://doi.org/10.1101/2021.04.16.440202; this version posted April 17, 2021. The copyright holder for this preprint (which was not certified by peer review) is the author/funder, who has granted bioRxiv a license to display the preprint in perpetuity. It is made available under aCC-BY-NC-ND 4.0 International license.

a

dCas9

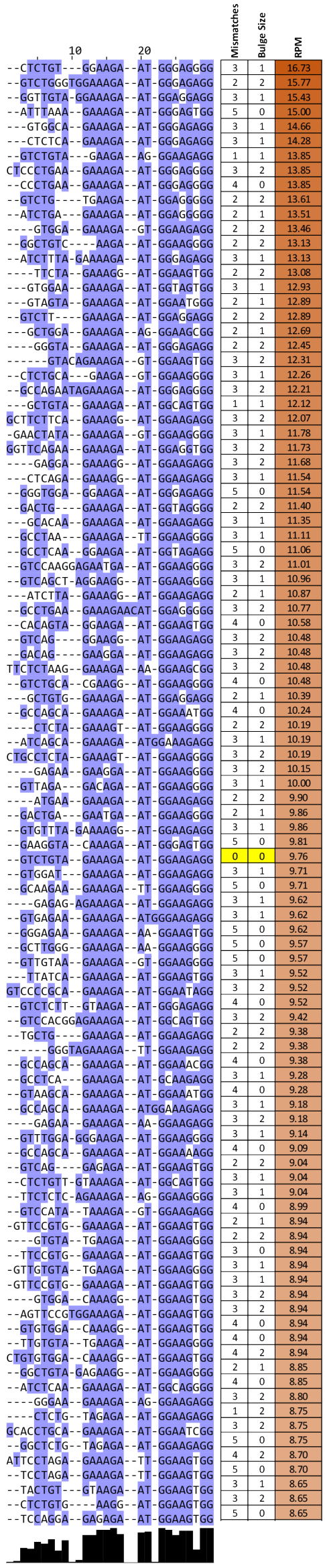

b

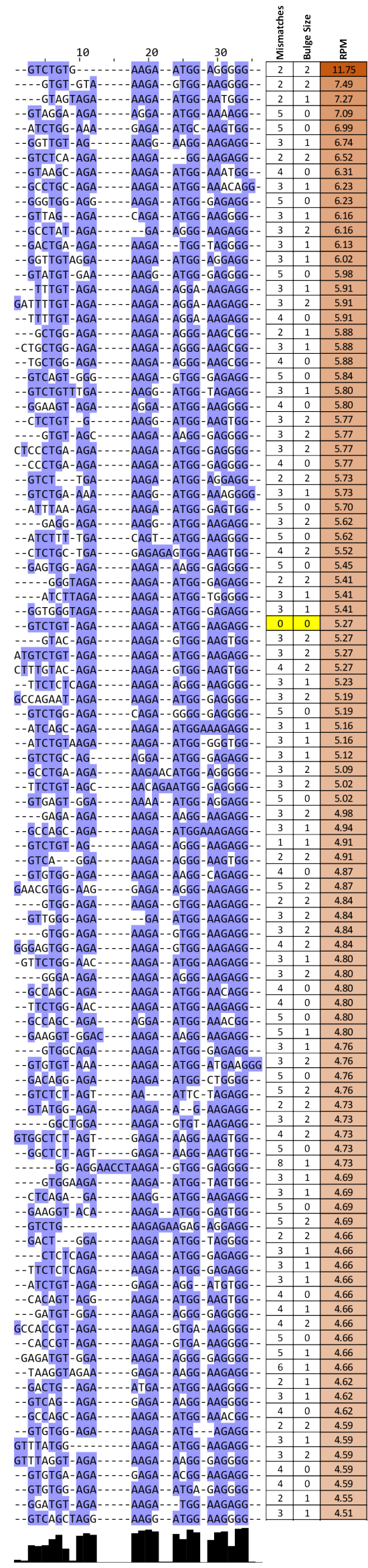

Supplementary Figure 10: Multiple sequence alignment of offtarget sites identified by in vitro dCas9 and Cas9 CasKAS for the "Nanog-sg3" sgRNA. Shown are the top 100 off-target sites as predicted by Cas-OFFinder and ranked by CasKAS signal. The on-target site (if within the top 100) is highlighted in yellow. 
bioRxiv preprint doi: https://doi.org/10.1101/2021.04.16.440202; this version posted April 17, 2021. The copyright holder for this preprint (which was not certified by peer review) is the author/funder, who has granted bioRxiv a license to display the preprint in perpetuity. It is made available under aCC-BY-NC-ND 4.0 International license.

a

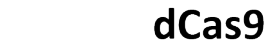

10
10
20
-..-TAGGAAGGGA-AAG-AAGAAAGG GAAGGAAAGAAGGAAG-AGGAAAGG - GAAAGCAAAAAGGAAG-AAGGAAGG - GAGGGAGGAAAGAAAG-AAGGAAGG - GAAGGAATGG-AAG-AAGAAAGG - AAGAAGGAATGG-AAG-AAGAAAGG - - GAAAGGAAGG-AAG-AAAAAAGG - gaAGgagaGa-AGG-AAGAAAGG - GAAGGAGAGA-AGG-AAGAAAGG -..-GAGAGGGAAG-AAG-AAGGAAG -AAGAGAGGGAAG-AAG-AAGGAAGG - GGGAGGGAGGAA-AGG-AAGAAAGG GTGGGAGGGAGGAA-AGG-AAGAAAGG - GAATAGGAGGAAGAAG-AAGAAGGG -- GAGTGAGAGAAGGAAG-TAAAGAGG GAGAGAAAGAAGTG-AAG-AGGAAAGG - GAAAGAGGGA-AAG-AAGGAAG GAGAAGGAAGTA-AAG-AGGAAAG GACAGAAGGAAGGAGG - AAGAAAGG - GAAAAAGAGG - AAG-AAGAAAGG GAGGAAGAGAGA-A--- AAGAAGG -gagtGGGaGCAGgaga-ATGAGAG - GAGGAAGAGAGG-AAGAAA GAGGGAGGGAGAAA-GTG-AGGAAGG -GAGACAGAGAG--AGG-AAGAGAGG - GAGACA-GACAG-AgG-AAGAGAGG GAGGAGAAGGAG-AAG-AAGAAAGG GAGACGGAGG -- -AAG-AGAGAGG GAGACAGGGAGGAAGG-AAGAGAG GAAGGAAAGAAGGAAG-CATAATGG -AGGAAGGAAGAG-AGG-AAGAGGGAAGAGACAGAGGAA-GAA-AAGGAAG GAGAGGGAGAGAGG-AAG-AGAGAGG - GaGGGAGGAAAGAAAG- AAGAAAG GAGGAAGAGA- - -GAG-AGGAAGGG GAGGAAGAGAGGCAGG-AAGAGGG 促GGGAGAGGGGG-AAGGAAGG - GACACAGAGA-GAG-AGGGAGGG -AAGGAAGAAGAA-AAG-AAGGAAGG --GAGGGAGGGAGGCAGT-AAGAAAGG GAAGGAAGGAAGAG-AGA-AAGAAAGG - GTGAAGAAGG -- -AAG-AAGGAAGG GAGAGAGAGAGAGG-..-AAGAAAGG - -GAGAAGGAGAAG-AAG-AAGAAGGGAGACAAGGAAGTA-AAG-AAGAAAGG - -GAGACAGAAGGAGCAA-CAGAAAGG GAGACAAGGAAGTA-AAG-AAGAAAGG - - GAGAACAAGA-CAG-AAAAGAGG GACAAGGAAGTA-AAG-AAGAAAGG - GGGAGAGAGAGAGAGG-AAGAAAGG GAAGGAAAGAATAA-AAG-AAGGAAGG - - GAGGAGGAGAAG-GAG-AAGAAAGG ---GAGAGAGAGG-AGG-AGGAAAGG -AAGAAGGGAGAAA-AGG-AAGAAGGG - AAGACAGAAAAGAGGG-AAGgAAGg --ACTTCAAAGGAAGAAG-AGGAAAGG GAAGAAAGGAGGGA-AAG-AGGAAAGG GAGGGAAAGAGGAA-AGG-AAAGAAGG AAGGGAGAGAGAGG-AGG-AGGAAAGG
--AAGGGAGAGCAG-ATG-AAGAAGGG - AAGGGAGAGCAG-ATG-AAGAAGGG --GAATGAGGGAAGAAAG-AAGGAAGG GAGGGAAGGAGGGA-AAG-AAGAAAGG --GAGGAAGAGATGGAGAGAAGAAAGG -GAGACAGAGATG---G-AAGGAGGG -CAGGGAGAGA-GAG-AAGAAGGG -GAGAAAAAGG-AAG-GAGAGAGG -GAAGGAGGGA-AAG-AAGAAAGG -GAAGCAGAGAAGAAAG-GAGGGAG -AAGAAAGAAGAG-AAA-AAGAAGG GAGACAGGGAGAGA-GAG-AAGAAGGG GAGAAAGGGAGCAA-GGC-AAGAAGGG - - GAAGCAGAGA-AGA-AAGGAGGG AGAGAGAGAAG-AAG-GGAATCGG -GAGAGAGGGGAG-AAA-GAAAAAGG - GACAGGGAGAGA-GAG-AAGAAGGG AAAGAAGAGAAA-AAG-AAGgAaG - AAGAAAGAAGAG-AAA-AAGGAAGG -AAGGACGGAAGG-AAG-AAGGAAGG GAAGGAAGGAAGGA-AAG-AAGAAAGG GAAAGAAAGAAGAG-AAA-AAGGAAGG GAAAGAAAGAAGAG-AAA-AAGGAAGG GAAGGAAGGAAGGG-AAG-AAGGAAGG --GAAAGAGAGAGAAG-AAGAAGGG --GAAAGAGAGAGAAG-AAGAAGGG GAGAGAGAGGACAG-AGG-AAGAGGGG -GTGTCATGTACAGAAG-AGGAATGG - GTGTCATGTACAGAAG-AGGAATGG GAGAGAGGACAG-AGG-AAGAGGG GAGGGAGGGAAGGA-CAG-AAGAAGG GAGAGAGGACAG-AGG-AAGAGGG GGGAAAGAGAGAGAAG-AAGAAGGG GAGAGAGAGGACAG-AGG-AAGAGGG --GAGTCAGAGCAG-AAGATGTAATG -GAGGAAGAGCAG-AAG-AAGAGGG-AagATG - GAGGGAGAGG-AAG-AGGAAGG -AGGAGGGAGG --AAG-AAAAAAG
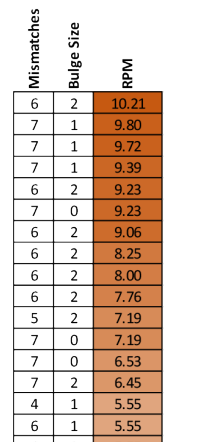

b

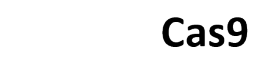

$\stackrel{10}{20} \stackrel{20}{10}$ AGAGAAAGAAGTGAAGAG - GAAAGG-GAAAGAGGGAAAGAA-GGAAGG- GAAAGGATGGAAGGA-GAAAGGGACAGAAGGAAGGAG - GAA-GAAAGG GAGACAAGGAAGTAAAGAA-GAAAGGGAAGAAAGGAGGGAAAGAG-GAAAGGGAGGGAAAGAGGAAAGGAA-AGAAGGTACACTGAGAAGAAACAG--GAAGGGGAGGGAGGGAAGGACAGAA-GAAGGG GAGGGAGTGAGGAAAGAAG-GAAGGGAAGGAAAGAAGGAAGGAG-GAAGG-- - - GAGGGAGAGGAAGAG-GAAGGG- TGTCATGTACAGAAGAG-GAATGG- GAGAAGGAGGGAGGA-GGATGG GAGGGAAGGAAGGAATGAA-GAAAGG GAGGGAAGGAAGGAATGAA-GAAAG GAGAGAGGGAAGGAAGAAG-GAAGG ---- GAAGGAGAGGAGGAG-GAAGG - TAAACAGAGgAGAAACACGAAGG - GAGGGAGGAAAGAAAGAA-GGAAGG G-AAAGCAAAAAGGAAGAA-GGAAGG GAGGGAGGGAAGAAGGAA- GAAAGG-..-TAGGAAGGGAAAGAA-GAAAGGGAAAAAGAGAAGGAAGGAG-GAAGG-GAGGGTGGGAAGAAAAGGA - GGAAGG ---AGGAAGGAGGAAGAA-GGAAGG GAAGGAAAGAAGGAA-GAG-GAAAGG- - AGGAAAGAGGAAGAA-GGAAGG - GAGGAGGAAGAAGAGAA- GATGG - GAAACAGGAAAGAGGAA-GAAGG - AAGATGgaggagaAAAA-AAGAGG ACTTCAAAGGAAGAAGAG-GAAAGG - GAAGGAGAGAAGGAA- GAAAGG - - GAGATAGAAAGGAAAGACGAAAGGGAGGGAGAGAAGAAAGAAG-GAAGG - - - - - GAGGGGAGAGAGAAGGAGA-GAAAGG- - AAGGCAGAGAGAGAAGA-GAAAGGG GAAAAGGAGGAAAAGAA-GAAGGG- - GAGGAGGATAAGGCA-GAAAGG -GAGATGGGAAAGGAGAA-TAAGGG - AAGGAGGAAAAGAAGAA-GGGAGG -AATAGAGAGGAAAAAAA-GAAAGG CAGGAGGAGGATAAGGCA-GAAAGG - GAGGGGAAAAGGAAAGAA-GGAGGGGAGAATAAGGGAAAAAAGA-AAAAGG -AAGTAGGAAGAAGAA-AAAAGG- - GAAGGAATGGAAGAA-GAAAGG -AAgAAGGAATGGAAGAA-GAAAGG- - GAGAGAGAGGGAGAA-CTGAGG - GAGGCAAAGAAGAAGAATGAAGGG GAGAAAGAGGAGAAA-GAAAGG - GAAAGAAAGGAAGAG-GAAGGG GGGAGGGAGGAAGAA-AGAGGG GAAGGAGAGAAGGAA-GAAAGG -GAGAAAGAAAGGAAGAG-GAAGGG- GAGAAAGAAAGGAAGAG-GAAGGG-GAGGGAGGGAGGAAGAA-AGAGGG- AAGGAAGAGAGAAAAGAA-GGAGGG -GAGGCGGCAGAAGAA-GAAAGG-AAGTTGGAGAAGATGGA-GGAGG- -AAGTTGGAGAAGATGGA-GGAGG-GAGGAAGGGAAGGAA-GGAAGGGAAGGAGAGAGGGAAGGAG-GAAGG-GAGGGAAGGAGGAAGGGAA-GGAAGG-GAGGAAGAGAAGAAGAA-GACAGG- - GAGAAAAAGGAAGAG-GAAGGG --GAGAAAAAGGAAGAG-GAAGGG-GAGGGAGGAAAGAAAGAA-GAAAGGGAGGGAGAGAGGGAGGAAG-GAAGG - GAGAGAAAAAGGAAGAG-GAAGGG- GAGACGGAGGAAGAG-AGAGGG GAGAGAGAGAATGAAGAG-AGAGGG - - GAATACAAGAAGGAA-GGAGGG -GAATACAAGAAGGAA-GGAGG GAGAAGGGAAGGGGAAGGA-GAAGGGAGAAGGGAAGGGGAAGGA-GAAGG -..-GAAGGAGAGAAGGAA-GGAAG -GAGAACGAAGGAGGGAA-GGAAGG -GAGGAGGAGGGAATGAA-GGAAG AAGGAAGAAGAAAAGAA-GGAAG AAGAAAGAAAAGAAAGAA-GGCAG GAGGGAAGGAGGGAATGAA-GGAAG GAGGGAAGGAGGGAATGAA-GGAAGGGAAGGTGGGAACCAGAGAA-GGAAG GAAGGAAGGAAGAGAGAAA-GAAAGGG-GAAAAGAAAAGAAGGAA-GGAAGG GAGAGAGAGA- - - GAGGAA-GAAAGG - GAGAGGGAAGAAGAA-GGAAGG ---GAGAGGGAAGAAGAA-GGAAGG GAGGAAGGGAGAAAAGGAA-GAAGGG- GGGAGGGAGGAAAGGAA-GAAAGAAGAGAGGGAAGAAGAA-GGAAGGAAGAGAGGGAAGAAGAA- GGAAGG-GAATAGGAGGAGGATAA-GGGGG GACAAGGGAAGGAGAAGGA-GAAGGG -

- - GAATAGGAGGAGGAT-AAGGG

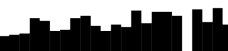

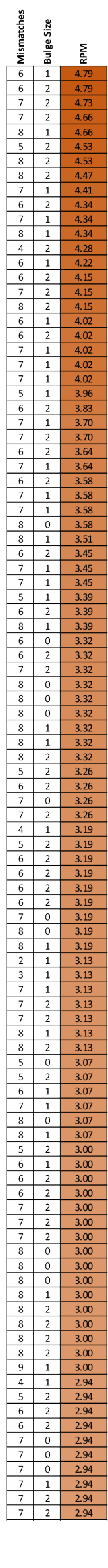

Supplementary Figure 11: Multiple sequence alignment of offtarget sites identified by in vitro dCas9 and Cas9 CasKAS for the "EMX1_Tsai" sgRNA. Shown are the top 100 off-target sites as predicted by Cas-OFFinder and ranked by CasKAS signal. The ontarget site (if within the top 100) is highlighted in yellow. 
bioRxiv preprint doi: https://doi.org/10.1101/2021.04.16.440202; this version posted April 17, 2021. The copyright holder for this preprint (which was not certified by peer review) is the author/funder, who has granted bioRxiv a license to display the preprint in perpetuity. It is made available under aCC-BY-NC-ND 4.0 International license.

a

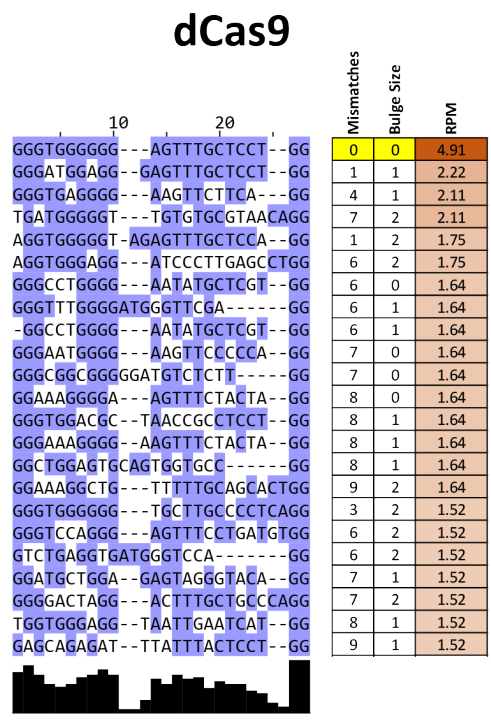

b

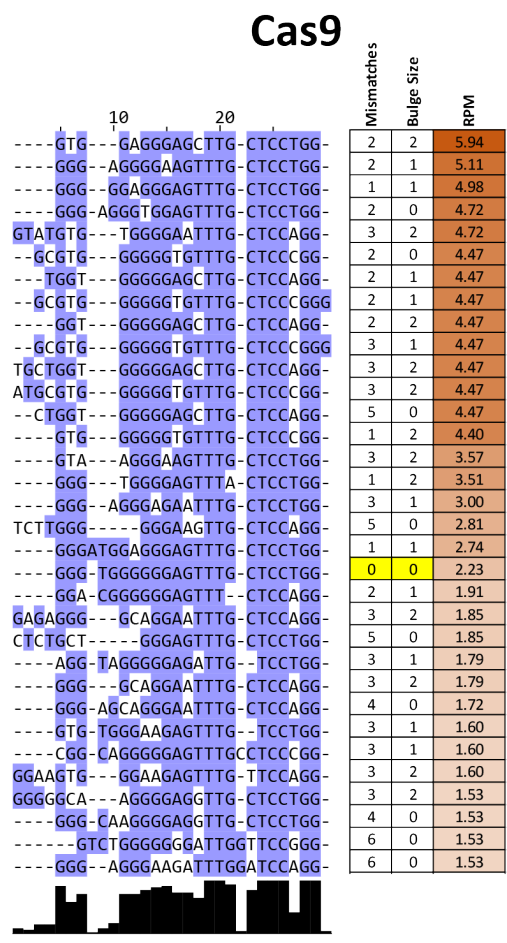

Supplementary Figure 12: Multiple sequence alignment of offtarget sites identified by in vitro dCas9 and Cas9 CasKAS for the "VEGFA-site1" sgRNA. Shown are the all target sites with RPM $\geq 1.5$ as predicted by Cas-OFFinder and ranked by CasKAS signal. The on-target site (if within the top 100) is highlighted in yellow. 

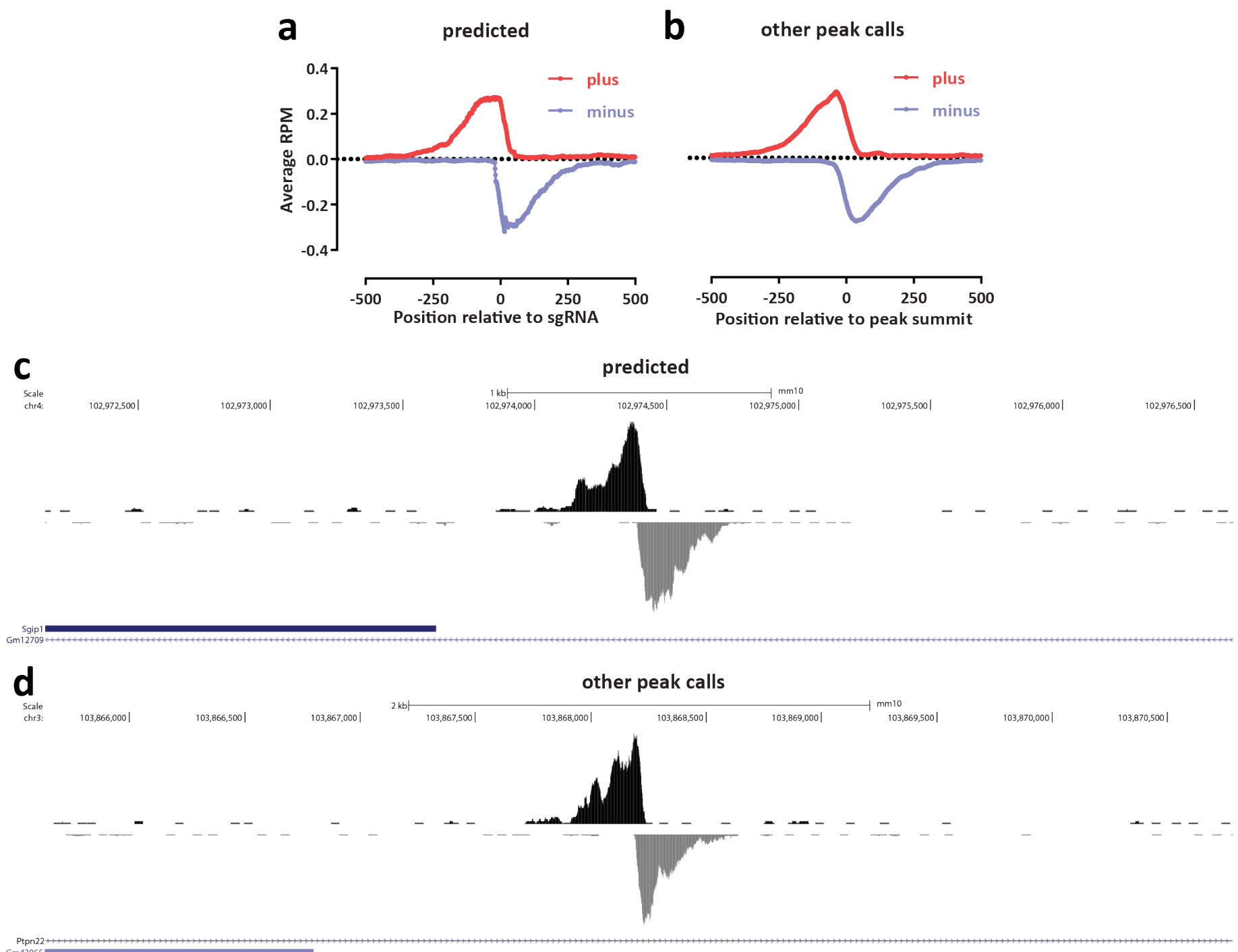

Supplementary Figure 13: CasKAS identifies proper off-target sites that are missed by sgRNA prediction algorithms. Shown is in vitro dCas9 CasKAS for the "sgRNA \#1" sgRNA. Peaks were called de novo using MACS2, then intersected with Cas-OFFinder off-target prediction, and the outersect was manually filtered to exclude obvious artifacts based on peak shape (e.g. arising from repetitive elements in the genome). (a) Aggregate forward- and reverse-strand profiles around off-target sites predicted by Cas-OFFinder (centered on the sgRNA); (b) Aggregate forward- and reversestrand profiles around sites not predicted by Cas-OFFinder (centered on the MACS2 peak summit); (c) Example UCSC Genome Browser snapshot of a CasKAS read profile around an off-target site predicted by Cas-OFFinder; (c) Example UCSC Genome Browser snapshot of a CasKAS read profile around an off-target site not predicted by Cas-OFFinder. Both predicted and identified through peak calling sites exhibit the expected asymmetric read distribution around a fixed occupancy point (the sgRNA-dCas9 RNP complexed with DNA). 
bioRxiv preprint doi: https://doi.org/10.1101/2021.04.16.440202; this version posted April 17, 2021. The copyright holder for this preprint (which was not certified by peer review) is the author/funder, who has granted bioRxiv a license to display the preprint in perpetuity. It is made available under aCC-BY-NC-ND 4.0 International license.

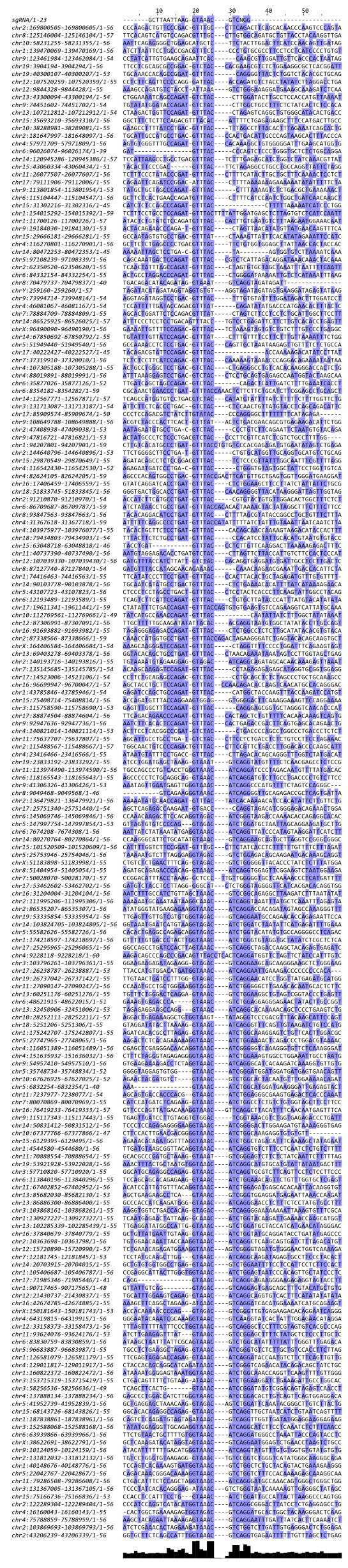

Supplementary Figure 14: Multiple sequence alignment of off-target sites identified by in vitro dCas9 and Cas9 CasKAS for the "sgRNA \#1" sgRNA outside the list of predicted off-targets by CassOFFinder. MACS2 peak calls were manually filtered to exclude artifactual peaks, then the sequence of the \pm 50 -bp region around the peak summit was used as input to the multiple sequence alignment, together with the sgRNA itself. 
bioRxiv preprint doi: https://doi.org/10.1101/2021.04.16.440202; this version posted April 17, 2021. The copyright holder for this preprint (which was not certified by peer review) is the author/funder, who has granted bioRxiv a license to display the preprint in perpetuity. It is made available under aCC-BY-NC-ND 4.0 International license.

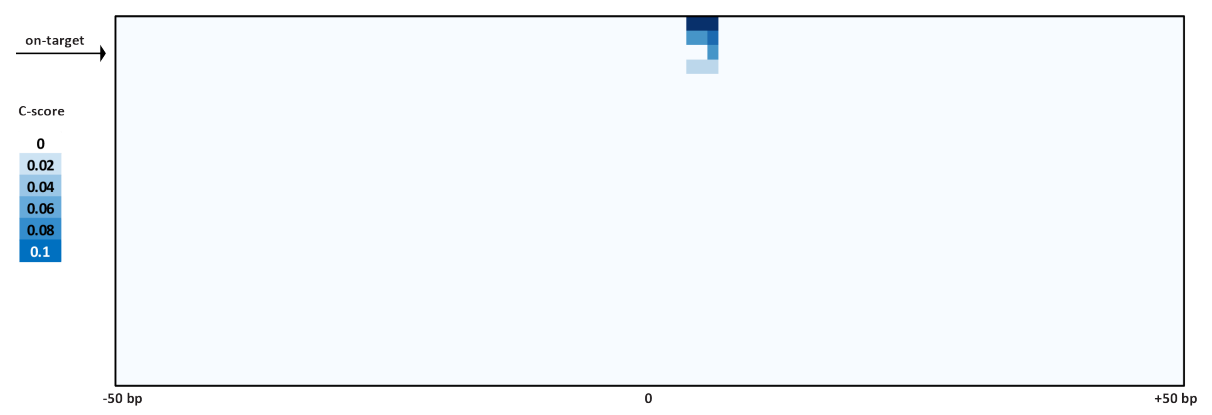

Supplementary Figure 15: Cutting profiles around on- and off-target sites for the VEGFA sgRNA. Four sites where cleavage is observed are identified within the list of predicted off-targets.

a

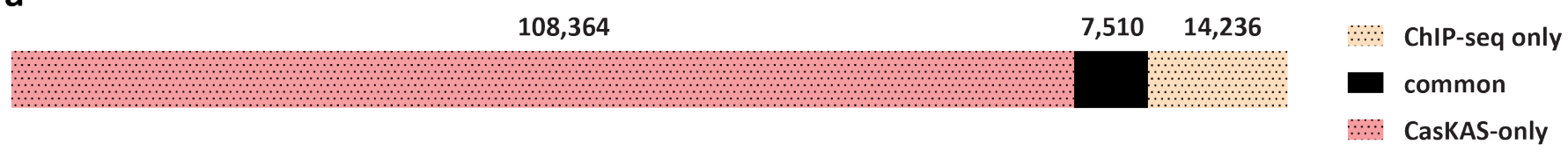

b

67,127

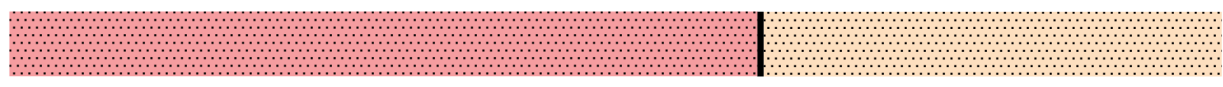

C

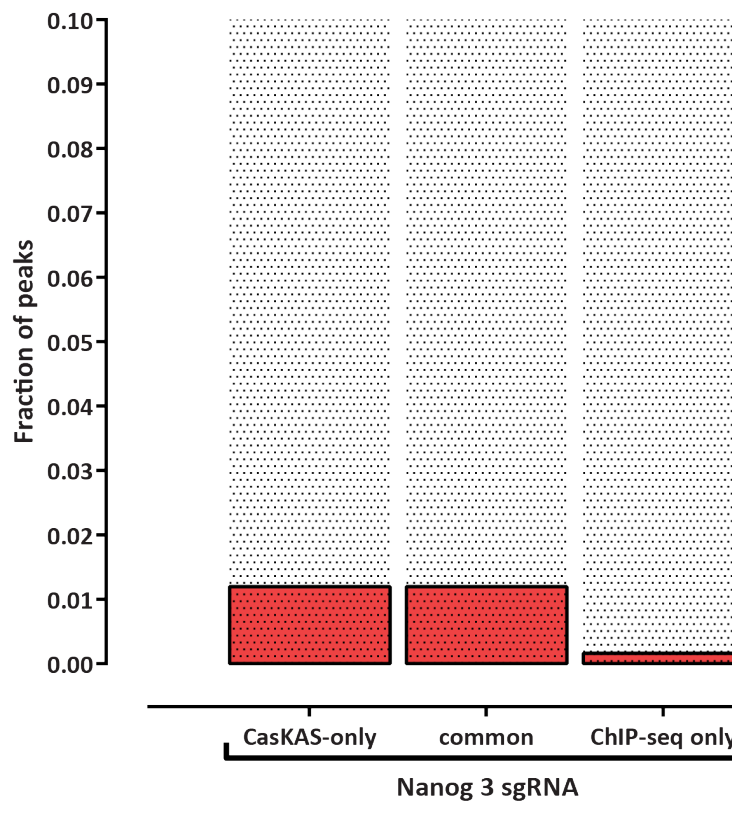

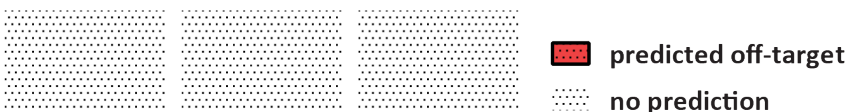

no prediction

CHANGE-seq only

common

CasKAS-only

Supplementary Figure 16: Comparing in vitro dCas9 results to using ChIP-seq and CHANGE-seq for off-target profiling. Shown is the overlap between MACS2 peak calls for the Nanog-sg3 sgRNA with Nanog ChIP-seq dataset (SRR1168384 from GEO accession ID GSE54745) in (a) and the EMX1 sgRNA with EMX1 CHANGE-seq (SRA accession SRX8227890) in (b). The fraction of peaks common or unique to each assay that are predicted to be off-targets for each sgRNA by Cas-OFFinder is shown in (c). 

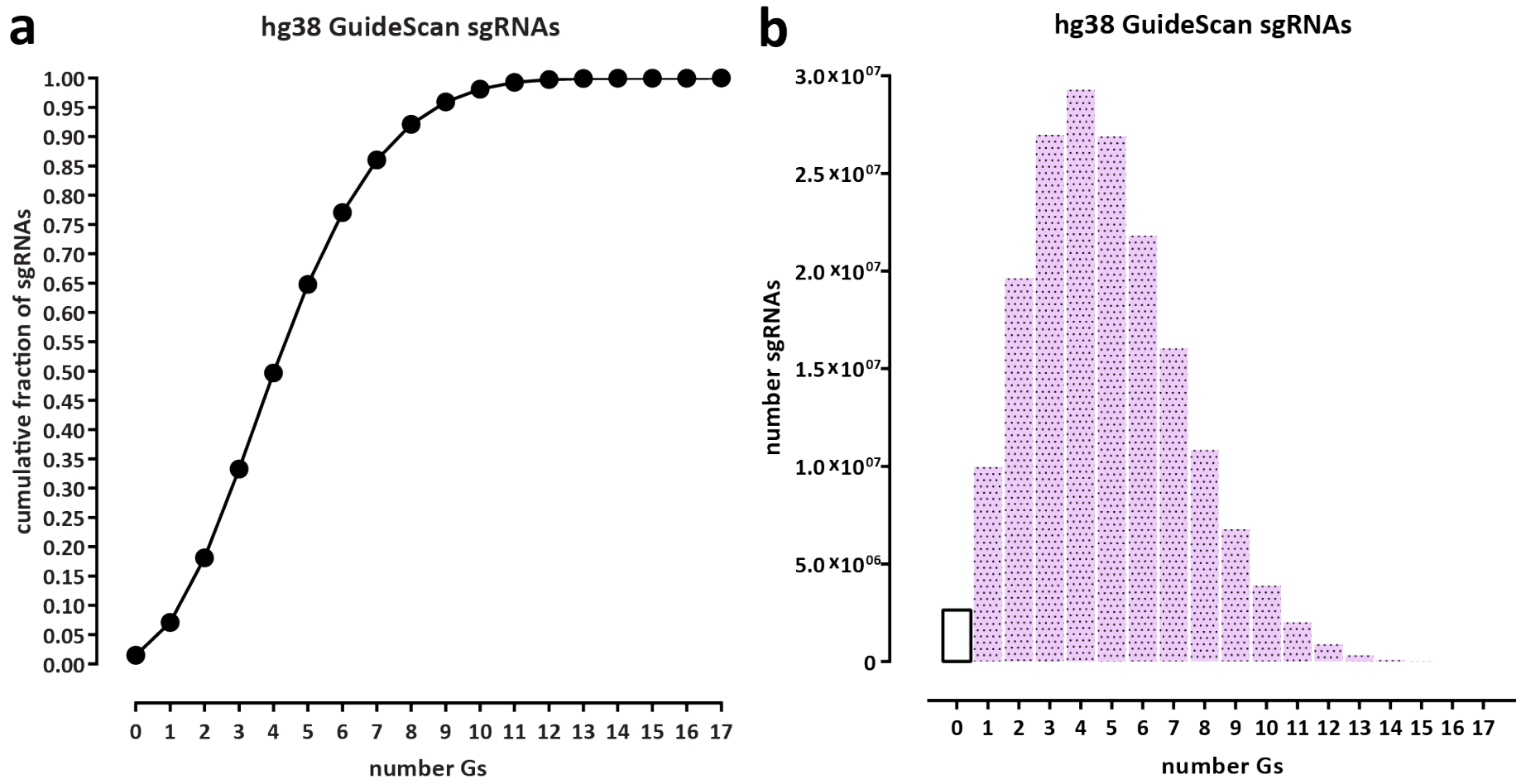

Supplementary Figure 17: Most sgRNAs in the human genome contain multiple G nucleotides and are thus subject to labeling by $\mathbf{N}_{3}$-kethoxal. Statistics were calculated for all valid sgRNAs as defined by GuideScan 18 (a) Cumulative fraction of sgRNAs. (b) Absolute number of sgRNAs.

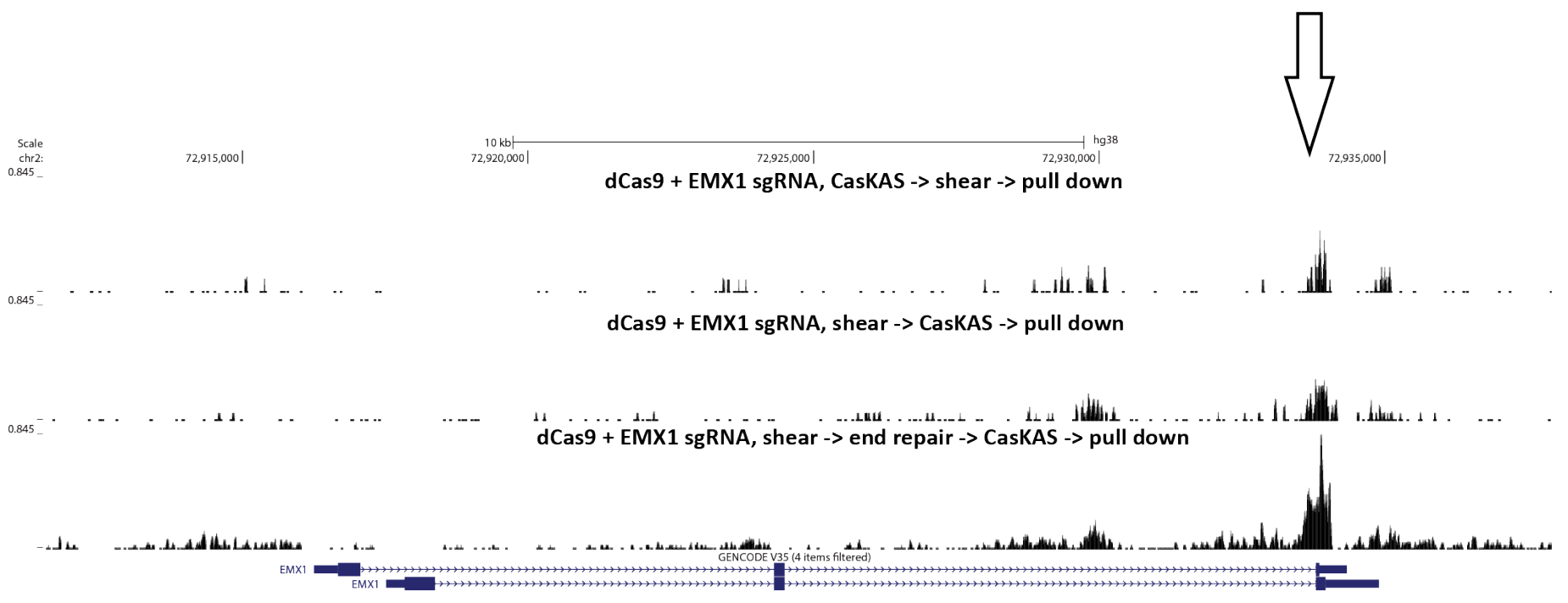

Supplementary Figure 18: CasKAS can be performed on pre-sheared DNA. CasKAS was performed in vitro using the EMX1 sgRNA, first, conventionally, by carrying out the CasKAS reaction, then isolating and shearing genomic DNA, and also by pre-shearing the DNA and carrying out the CasKAS reaction on the fragmented DNA. The concern in that case is that the presence of sticky ends containing Gs and unprotected from the action of the $\mathrm{N}_{3}$-kethoxal would lower the background. This problem can be addressed by carrying out end repair on the sheared DNA prior to the CasKAS reaction. 